P A R T V BIOLOGICAL APPLICATIONS 

C H A T E R 12

\title{
SYNCHROTRON RADIATION- BASED NUCLEAR RESONANT SCATTERING: APPLICATIONS TO BIOINORGANIC CHEMISTRY
}

\author{
Yisong Guo, ${ }^{1}$ Yoshitaka Yoda, ${ }^{2}$ Xiaowe Zhang, ${ }^{3}$ Yuming Xiao, ${ }^{4}$ and Stephen P. Cramer ${ }^{5}$ \\ 'Department of Chemistry, Carnegie Mellon University, Pittsburgh, PA, USA \\ ${ }^{2}$ Research and Utilization Division, SPring-8/JASRI, Kouto, Sayo, Hyogo, Japan \\ ${ }^{3}$ Photon Factory, KEK, I-I Oho, Tsukuba, Ibaraki, Japan \\ ${ }^{4}$ HPCAT, Advanced Photon Source, Argonne National Laboratory, Argonne, IL, USA \\ ${ }^{5}$ Department of Applied Science, University of California-Davis, Davis, CA, USA
}

\subsection{INTRODUCTION}

Mössbauer spectroscopy is a powerful tool for a broad range of scientific and technological applications. Among them, the application of Mössbauer spectroscopy to bioinorganic chemistry has achieved a great success in elucidating the geometric and electronic structures of iron centers found in metalloproteins through the detection of the hyperfine interactions between ${ }^{57} \mathrm{Fe}$ nuclei and the surrounding electrons [1-4]. The information derived from Mössbauer spectroscopy is crucial for the understanding of the chemical and biological processes performed by these proteins. However, the intensity of the Mössbauer effect depends on the Lamb-Mössbauer factor fLM, the probability of observing recoilless emission or absorption of nuclear resonant gamma radiation. This makes the application of this technique to nuclei with small $f_{\mathrm{LM}}$, such as ${ }^{61} \mathrm{Ni}$, less successful. To overcome the dependence of $f_{\mathrm{LM}}$, an alternative technique is the method of time differential perturbed angular correlations (TDPACs) [5], which does not depend on the binding state of the probed nuclei, therefore, not depend on $f_{L M}$. TDPAC has been used in a large amount of bioinorganic applications to probe the metal binding site geometry and illustrate the relation between the protein function and the structure and dynamics of the metal binding site [6,7]. Both Mössbauer spectroscopy and TDPAC rely on suitable radioactive sources to provide nuclear resonant gamma radiations. In Mössbauer, radioactive sources generating single line nuclear resonant gamma radiations with relatively long source lifetime are needed. The contribution of both source and sample constrains the best achievable spectral resolution in Mössbauer spectroscopy to be $2 \Gamma$ ( $\Gamma$ is the natural linewidth of nuclear transition, which is $4.66 \mathrm{neV}$ or $\sim 0.1 \mathrm{~mm} \mathrm{~s}^{-1}$ for ${ }^{57} \mathrm{Fe}$ ). In TDPAC, more complicated demands for the radioactive source, in particular the necessity of a suitable nuclear decay cascade, make TDPAC not applicable to most of Mössbauer isotopes. Furthermore, it is necessary to enrich protein metal sites with radioactive sources, and the aftereffect from the decay of the parent isotope can further complicate the data collection and data analysis [7]. Therefore, the development of radioactive source-free techniques is crucial for the future applications of Mössbauer spectroscopy and TDPAC to bioinorganic chemistry.

Mössbauer Spectroscopy: Applications in Chemistry, Biology, and Nanotechnology, First Edition.

Edited by Virender K. Sharma, Göstar Klingelhöfer, and Tetsuaki Nishida.

(c) 2013 John Wiley \& Sons, Inc. Published 2013 by John Wiley \& Sons, Inc. 
The use of the third-generation synchrotron radiation (SR) source overcomes the limitations imposed by radioactive sources mentioned above. The high brightness, large-range energy tunability, and highly linear polarized and collimated beam with very small beam size make SR a superb radiation source for Mössbauer spectroscopy and TDPAC. Mössbauerlike information spectroscopy is usually obtained by a technique called nuclear forward scattering (NFS) [8]. There is also a growing number of examples of "true" synchrotron Mössbauer experiments, in which the synchrotron radiation is used to pump a single line source, which is then used for further experiments [9-II]. Finally, the SR variant of TDPAC is called synchrotron radiation-based perturbed angular correlations (SRPAC) [12]. All of these fall under the general umbrella of SR-based nuclear resonant scattering (NRS) techniques [13, I4].

Different from conventional Mössbauer spectroscopy, which is an energy-domain technique, NFS is a time-domain technique-it monitors the change of the nuclear decay signal from the nuclear excited states as a function of time. The use of SR in a time-domain approach eliminates the source contribution to the spectral linewidth, making the spectral resolution of NFS higher than the conventional Mössbauer. NFS has been applied to many different Mössbauer isotopes, and has been demonstrated as a promising new technique for studies in solid-state physics, materials science, geosciences, thin film, and bioinorganic chemistry [13-16].

SRPAC can be considered as a scattering variant of TDPAC [12]. Instead of populating the intermediate nuclear level via a cascade of preceding nuclear transitions after decay of the radioactive parent in TDPAC, the intermediate nuclear level is populated from below-the ground nuclear state-during incoherent, single nucleus resonant scattering of SR [12]. Directional selection and timing by the first detector in TDPAC are replaced in SRPAC by the direction and the timing of the incident SR. In both methods, the nuclear decay signal from the interference of indistinguishable paths from an intermediate nuclear level splitting by magnetic dipole and/or electric quadrupole interactions allows one to investigate hyperfine interactions and spin dynamics. The use of SR enables SRPAC to be applied to all nuclei with isomeric state and resonance energies achievable by SR. Since SRPAC signal is independent of the Lamb-Mössbauer factor, $f_{L M}$, it has been demonstrated to study electric quadrupole interactions and relaxation in soft condensed matter [12] containing Mössbauer isotope ${ }^{57} \mathrm{Fe}$ and ${ }^{119} \mathrm{Sn}$ [17]. It has also been demonstrated to study magnetic dipole interactions of a ${ }^{61} \mathrm{Ni}$-enriched $\mathrm{Ni}$ foil [18].

In this chapter, the application of ${ }^{57} \mathrm{Fe}$ NFS, ${ }^{57} \mathrm{Fe}$ SRPAC, and ${ }^{61} \mathrm{Ni}$ SRPAC to bioinorganic chemistry will be presented. The unique features of these two SR-based spectroscopic techniques will be discussed. The future development and prospect of these two techniques will be given.

\subsection{TECHNICAL BACKGROUND}

As mentioned in Section 12.I, Mössbauer, TDPAC, as well as SR-based NFS and SRPAC can all be used to elucidate the geometric and electronic structures of the local environment surrounding the probed nuclei through the detection of the energy splittings of the sublevels of nuclear excited states and ground states due to the hyperfine interactions between the nuclei and the surrounding electrons. There are three types of hyperfine interactions: isomer shift, quadrupole splitting, and magnetic splitting. Although, similar information can be obtained by using the spectroscopic techniques mentioned above, the SR-based techniques have unique features that differ significantly from the laboratory-based techniques. In the following, the theoretical and experimental aspects of NFS and SRPAC are introduced.

\subsubsection{Theoretical Aspects of NFS}

NFS is a time-domain Mössbauer spectroscopy using synchrotron radiation (see Fig. 12.I for the comparison between conventional Mössbauer spectroscopy and NFS). It is an elastic coherent scattering process, where different nuclear transitions are excited in phase simultaneously by a short X-ray pulse with sufficient energy bandwidth. Shortly after the $X$-ray pulse, the de-excitations of all the nuclear excited states begin also in phase, but soon the increasing phase difference leads to destructive interference and again to a constructive interference. This unique behavior, which is due to the energy differences of the sublevels of nuclear ground states and excited states caused by hyperfine interactions, is the basic process that leads to oscillations (quantum beats, QB) in the nuclear decay of forward-scattered radiation. In the following, we will discuss some special cases to further illustrate the NFS spectral features (for detailed discussion of the theoretical model, one should refer to Refs. 8,|9-2I).

12.2.1.1 Magnetic Hyperfine Interactions First, we discuss the NFS intensity in the presence of magnetic hyperfine interactions. We assume that the direction of the internal magnetic field in the sample is perpendicular to both 

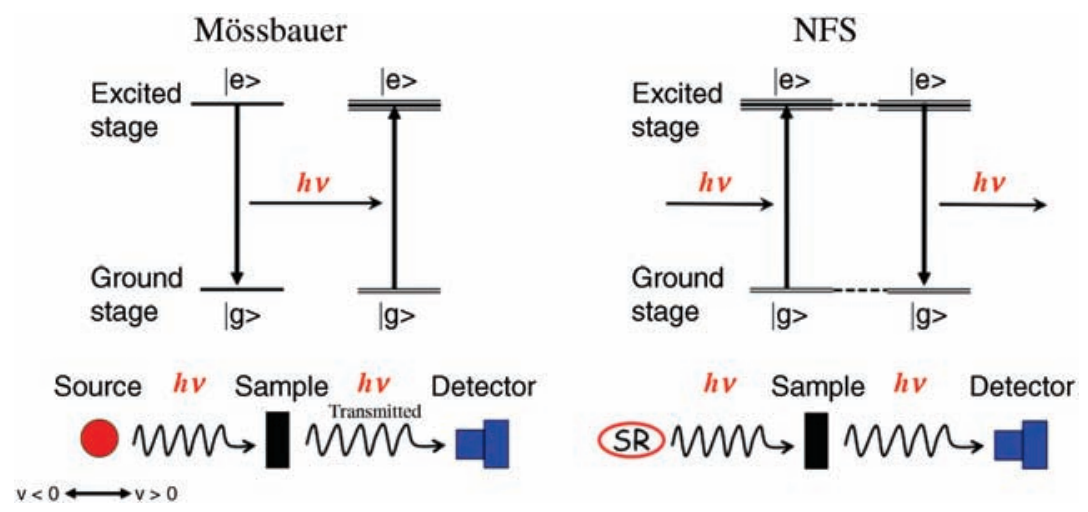

\section{FIGURE 12.1}

A schematic of the principles and experimental setups for conventional Mössbauer spectroscopy (left) and SR-based NFS (right). In conventional Mössbauer spectroscopy, a $\gamma$-ray is generated by a radioactive source $\left({ }^{57} \mathrm{Co}\right.$ for ${ }^{57} \mathrm{Fe}$ Mössbauer). A driver system is attached to the source to provide a Doppler shift to the energy to the emitted $\gamma$-ray. This $\gamma$-ray can be resonantly absorbed in the sample. The transmitted $\gamma$-ray intensity is registered in the detector as a function of Doppler velocity. In SR-based NFS, millielectron volt bandwidth $\gamma$ radiation is provided by synchrotron radiation and subsequent monochromators. This pulse coherently excites different nuclear transitions in the sample. The forward-scattered signal generated from the nuclear excited states is registered in the detector placed in the forward direction as a function of time.

the direction and the electric polarization of the incident SR (Fig. 12.2), which can be achieved experimentally by, for example, applying a small external magnetic field along this direction to magnetize a ${ }^{57} \mathrm{Fe}$ foil. Then only $\Delta m=m_{\mathrm{e}}-$ $m_{\mathrm{g}}=0$ transitions are allowed (Fig. 12.2), and the delayed intensity (NFS intensity) per time can be described as [8]

$$
\frac{\mathrm{d} I}{\mathrm{~d} t}=I_{0} \frac{t_{\text {eff }}^{2}}{16 \tau} \exp (-t / \tau) \cos ^{2} \omega t
$$

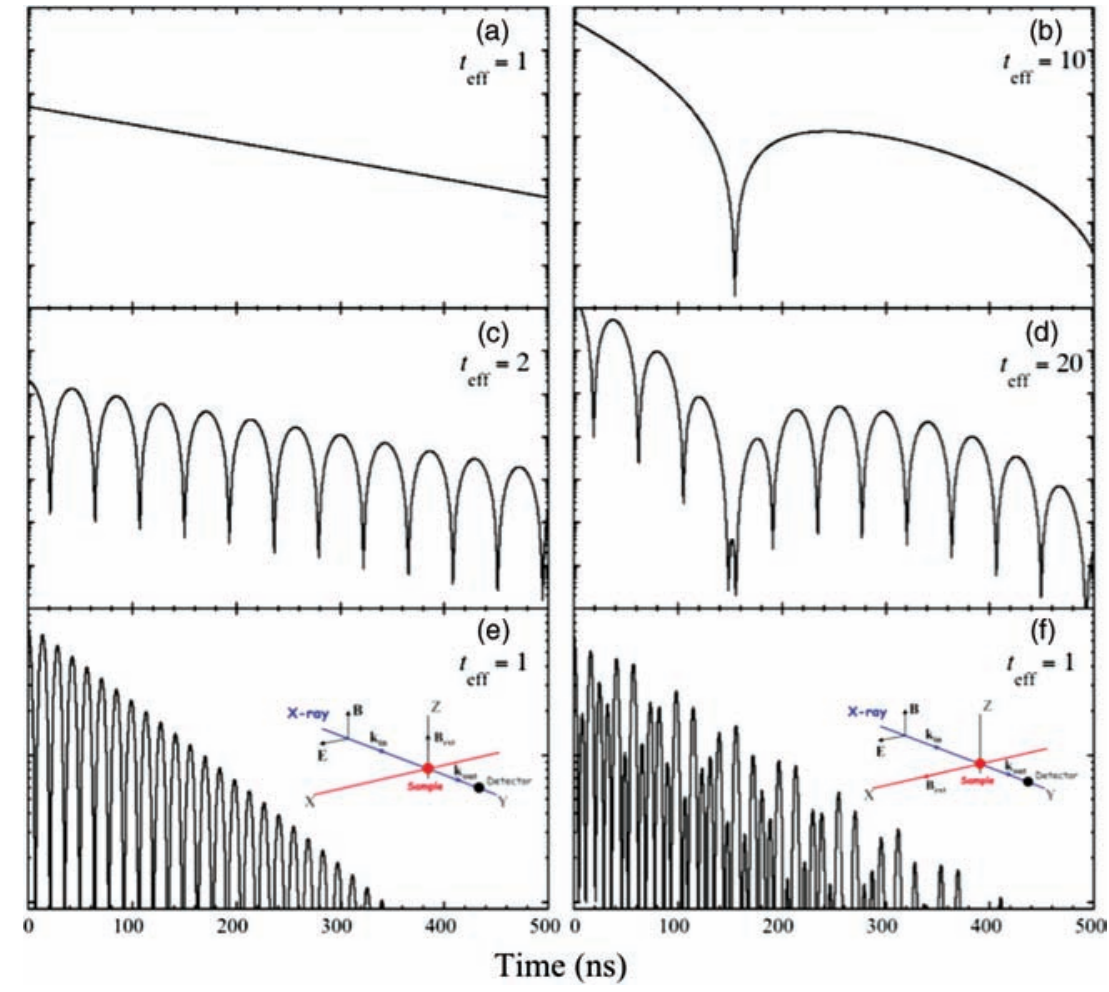

\section{FIGURE 12.2}

Theoretical NFS spectra for no hyperfine interactions with (a) thin absorber or with (b) thick absorber; single quadrupole splitting with (c) thin absorber or with (d) thick absorber; magnetic splitting with applied magnetic field $B_{\text {ext }}$ (e) parallel to magnetic field $\mathbf{B}$ of the incoming beam; and (f) magnetic splitting with applied magnetic field $\mathbf{B}_{\text {ext }}$ parallel to electric field $\mathrm{E}$ of the incoming beam. Insets show the scattering geometry. The effective thickness used is indicated in each figure. 
where $\hbar \omega=B\left(\mu_{\mathrm{g}}-\mu_{\mathrm{e}} / 3\right)$ is half the difference of $\Delta m=0$ transition energies. $t_{\text {eff }}=\rho \sigma f D$, is the effective thickness of the absorber (sample), where $D$ is the physical thickness of the absorber, $f$ is the Lamb-Mössbauer factor, $\sigma$ is the nuclear resonance cross section, for ${ }^{57} \mathrm{Fe}$, it is $2.56 \times 10^{-18} \mathrm{~cm}^{2}$. $\rho$ is the number of resonant nuclei per cubic centimeter. In this case, the NFS spectrum shows a single oscillation frequency (Fig. 12.2).

Next, we assume the direction of the internal magnetic field in the sample is parallel to the direction of the electric polarization of the incident SR (Fig. 12.2). This corresponds to the nuclear transitions of $\Delta m= \pm$ I (Fig. I2.2). The time spectrum now is given by [8]

$$
\frac{\mathrm{d} I}{\mathrm{~d} t}=I_{0} \frac{t_{\mathrm{eff}}^{2}}{16 \tau} \exp (-t / \tau)\left(\frac{3}{4} \cos \omega_{1} t+\frac{1}{4} \cos \omega_{2} t\right)^{2}
$$

Here, $\hbar \omega_{1}=B\left(\mu_{\mathrm{g}}-\mu_{\mathrm{e}}\right)$ is half the difference of the $-\mathrm{I} / 2 \rightarrow-3 / 2$ and $\mathrm{I} / 2 \rightarrow 3 / 2$ transition energies, and $\hbar \omega_{2}=$ $\mathrm{B}\left(\mu_{\mathrm{g}}+\mu_{\mathrm{e}} / 3\right)$ is half the difference of the $-\mathrm{I} / 2 \rightarrow \mathrm{I} / 2$ and $\mathrm{I} / 2 \rightarrow-\mathrm{I} / 2$ transition energies. Now there are two frequencies modulating the nuclear decay (Fig. 12.2).

12.2.1.2 Electric Hyperfine Interactions Finally we discuss the oscillations of time spectra caused by the nuclear ground-state and excited-state splittings due to electric field gradients (EFGs). We limited our discussion in an axially symmetric EFG (asymmetric parameter $\eta=0$ ), and furthermore, the EFG axis is randomly oriented over the space. Then we can get the time spectrum [8] (Fig. 12.2)

$$
\frac{\mathrm{d} I}{\mathrm{~d} t}=I_{0} \frac{t_{\mathrm{eff}}^{2}}{16 \tau} \exp (-t / \tau) \cos ^{2} \omega t
$$

with $\hbar \omega=\Delta / 2$, the half energy difference of the transition energies.

By inspecting Eqs. (I2.I)-(I2.3), one can find that NFS intensity is proportional to the square of the number of resonant nuclei in the sample and Lamb-Mössbauer factor $f$ due to the effective thickness of the absorber. Furthermore, in the absence of hyperfine interactions (the sublevels of the ground and excited nuclear states are degenerate), the NFS intensity reduces to a natural nuclear decay (Fig. 12.2).

Apart from the oscillations caused by nuclear hyperfine interactions, another oscillation due to multiple-scattering process of nuclear decay exists for the SR passing through a relative thick sample containing resonance nuclei. This oscillation is called dynamic beat (DB), it has been shown theoretically [22,23] and experimentally [24]. In the case of a single resonance (absence of nuclear hyperfine interactions), DB takes the form [22]

$$
A(t)=E_{0} \frac{t_{\text {eff }}}{\tau} \mathrm{e}^{-t / 2 \tau} \frac{J_{1}\left(2 \sqrt{t_{\text {eff }} t^{\prime}}\right)}{\sqrt{t_{\text {eff }} t^{\prime}}}
$$

where $t_{\text {eff }}$ is the effective thickness of the sample, $E_{0}$ is the nuclear resonance energy, $t^{\prime}=t / \tau$, and $J_{1}$ is the Bessel function of first kind and first order. $A(t)$ is the scattering amplitude, the intensity can be obtained as the square of the amplitude. From Eq. (12.4), we can summarize the characteristic features of DB: DB is not periodic, the apparent periods increase with time; the apparent DB periods decrease with increasing effective thickness $t_{\text {eff. }}$ The initial decay is speeded up proportionally to effective thickness $t_{\text {eff }}$.

Although DB is an interesting phenomenon, for spectroscopic measurements, it is mostly unwanted, since the beating pattern of DB will mix with QB to generate a complicated spectrum. Figure 12.2 shows some theoretical NFS spectra with different effective thicknesses. For more detailed discussion on DB, one can refer to Refs. 22,23.

\subsubsection{Theoretical Aspects of SRPAC}

SRPAC is another NRS technique that reveals nuclear hyperfine interactions by monitoring quantum beats superimposed on the nuclear decay signal. Instead of detecting the coherent scattering in the forward direction as in NFS, SRPAC detects the direct nuclear resonant fluorescence that can be observed in $4 \pi$ solid angle. (Note: The incoherent nuclear scattering is not the only channel of scattering that produces decay radiation into $4 \pi$ solid angle, the combination of NFS

and subsequent Rayleigh scattering can also direct the decay radiation into $4 \pi$ solid angle. Please see the discussion in Section 12.3.2.) SRPAC is an incoherent NRS, where the nuclei have different initial and final nuclear states during the 
scattering process. Therefore, it is possible, in principle, to identify the nucleus that scatters the photon, and thus incoherent NRS, such as SRPAC, can be considered as single nucleus scattering. The nuclear state change can happen by a change of spin of the ground state (spin-flip process) or by a change of the vibrational state of the nucleus (absorption/ emission of phonons). Coherent NRS, such as NFS, by contrast, is delocalized over the whole ensemble of nuclei, there is no change of state of any nucleus due to the scattering. So if a nuclear hyperfine interaction splits the sublevels of nuclear ground state and excited state, coherent NRS is sensitive to both ground-state and excited-state splittings, while incoherent NRS is only sensitive to excited-state splittings.

As mentioned above, SRPAC can happen with absorption or emission of phonons. If the energy bandwidth of the incoming beam is large enough to cover all the phonon-assisted processes, the SRPAC signal does not depend on

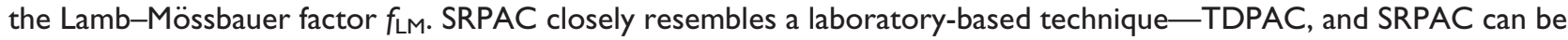
considered as a scattering variant of TDPAC. In both techniques, the angular correlation of $\gamma$ radiation is recorded. In a system with two $\gamma$ rays successively emitted from a nucleus through initial state, intermediate state and final state, the possibility of measuring the second $\gamma$-ray in a particular direction can have angular dependence with the direction of the first $\gamma$-ray when the first $\gamma$-ray is emitted anisotropically by an ensemble of oriented nuclear spins. Unperturbed angular correlation is characterized by the constant angular dependence between the two $\gamma$ rays as a function of time. If hyperfine interactions present, the nuclear spins can interact with them when the nucleus remains in the intermediate state for a finite lifetime. It will cause the precession of the nuclear spins and result in a perturbed angular correlation between the two $\gamma$ rays. As described in Refs. 12,25, in TDPAC, the intermediate nuclear level is populated from above via a cascade of preceding nuclear transitions after decay of the radioactive parent. In SRPAC, on the contrary, the level is excited from below - from the ground state. Directional selection and timing by the first detector in TDPAC are replaced in SRPAC by the direction and the timing of the incident SR. In both methods, the interference of indistinguishable paths from an intermediate nuclear level splitting by magnetic dipole and/or electric quadrupole interactions allows one to investigate hyperfine interactions and spin dynamics. A comparison drawing of their principles and experimental setups are shown in Fig. I 2.3 [12,25]. For more information on TDPAC, one can refer to Refs. 5,26 and for biological applications to Refs. 6,7.

A general expression of SRPAC intensity can be given as $[12,18,25]$

$$
I(t)=I_{0} \mathrm{e}^{-t / \tau_{0}}\left[1-2 A_{22} R(t)\right]
$$

TDPAC
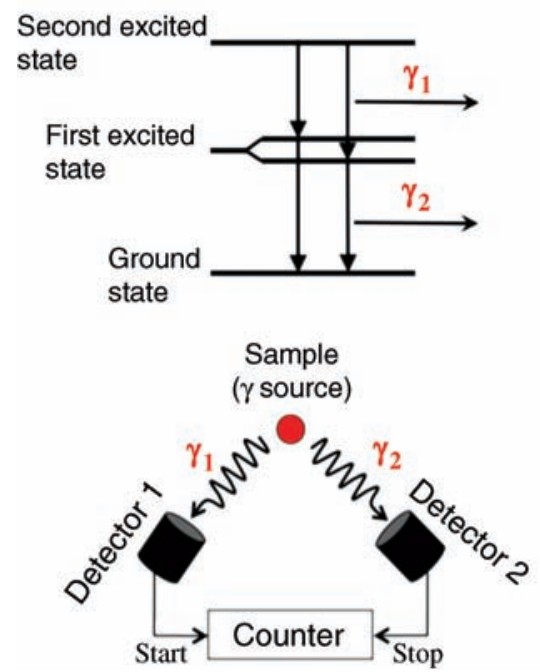

SRPAC
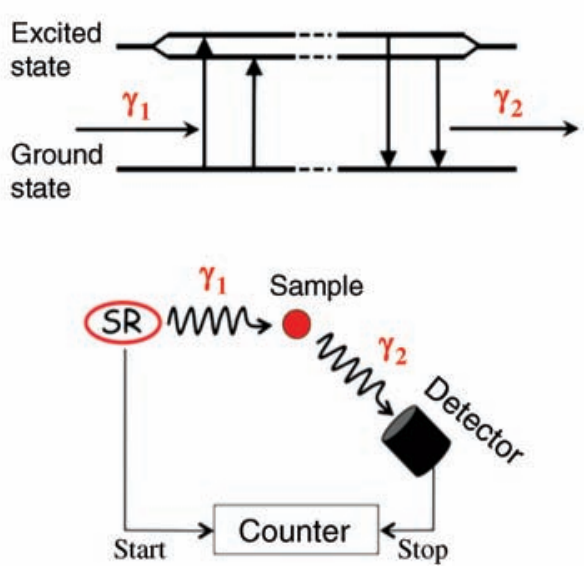

FIGURE 12.3

Schematic drawing of the principles and the experimental setup of TDPAC (left) and SRPAC (right). . In TDPAC, the intermediate nuclear level is populated from above via a cascade of preceding nuclear transitions after decay of the radioactive parent in the sample, which provides the timing "trigger" ( $\gamma_{1}$ on the left). The successive nuclear decay of the intermediate nuclear levels $\left(\gamma_{2}\right)$ and $\left(\gamma_{1}\right)$ are registered in two different detectors. In SRPAC, the level is excited from ground states through incident synchrotron radiation, which provides the timing trigger ( $\gamma_{1}$ on the right). The successive nuclear decay of the excited levels $\left(\gamma_{2}\right)$ is registered in the detector. Adapted from Ref. 12 


\section{FIGURE 12.4}

Top: (a) Theoretical curves of the SRPAC intensity for case of electric quadrupole interactions described in Eq. (12.6) for different polar angle $\theta$. The scattering geometry is shown on the bottom left; (b) theoretical curves of the SRPAC intensity for case of magnetic dipole interactions described in Eq. (12.7) (black curve) using the bottom left scattering geometry and Eq. (12.8) (grey curve) using the bottom right scattering geometry. Bottom: scattering geometries used in generating the curves shown on top.

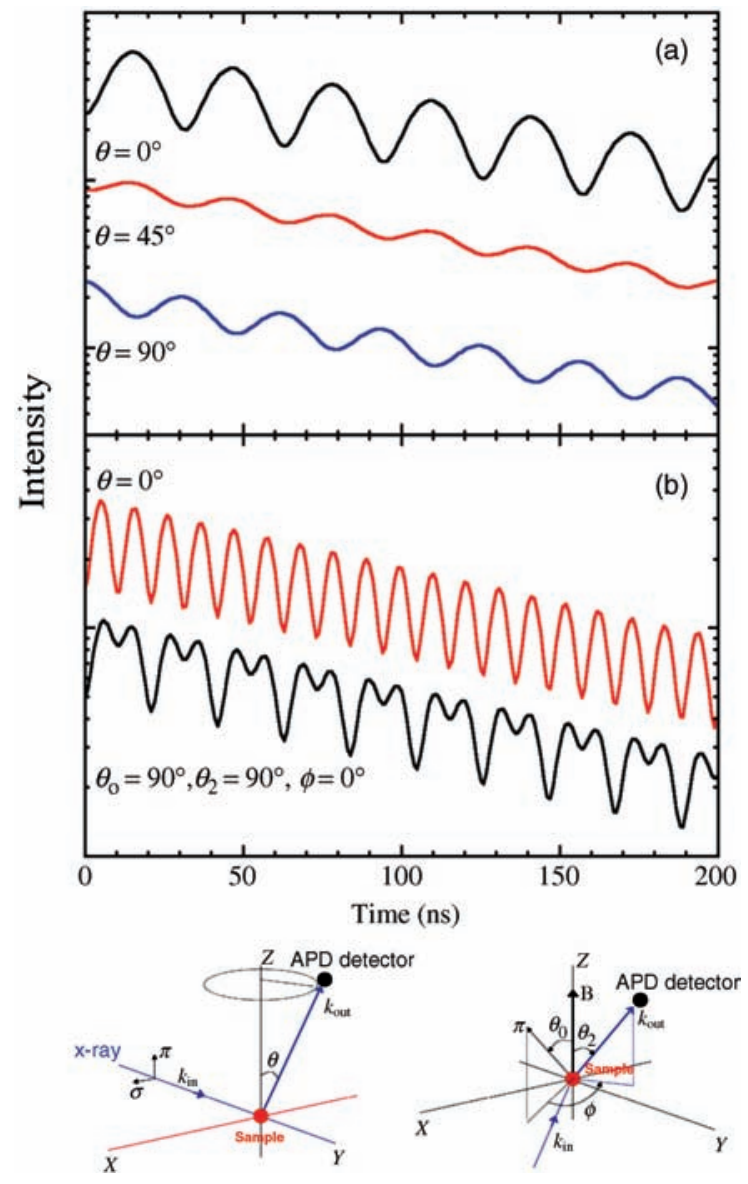

where $\tau_{0}$ is the lifetime of the nuclear excited state. $A_{22}$ is the anisotropy coefficient: for ${ }^{57} \mathrm{Fe}$, the value is 0.25 , and for ${ }^{61} \mathrm{Ni}$, the value is $0.14 . R(t)$ is the anisotropy factor, which, in some cases, can be factored into angular and time-dependent terms as $R(t)=G_{22}(t) P_{2}(\cos \theta)$. Here the angular dependence of the SRPAC intensity is described by a second-order Legendre polynominal $P_{2}(\cos \theta)$ and $\theta$ is the angle between the scattered photon $\mathbf{k}_{\text {out }}$ and the magnetic polarization of the incoming photon $\pi$ (Fig. 12.4). The time dependence of the SRPAC intensity is described by the perturbation factor $G_{22}(t)$. If there is no nuclear hyperfine interactions, $G_{22}(t)=I$, and the SRPAC intensity follows the natural decay. Next, we will discuss the theoretical description of the SRPAC intensity in the presence of nuclear hyperfine interactions.

12.2.2.1 Electric Hyperfine Interactions First, we will consider the SRPAC intensity with the presence of an axially symmetric quadrupole interaction caused by the interaction of the quadrupole moment of the excited nuclear state with an EFG produced by surrounding electrons and other nuclei. The direction of EFG is assumed to be isotropically distributed. In case of ${ }^{57} \mathrm{Fe}$, the excited-state spin $I=3 / 2$, then the SRPAC intensity can be expressed as $[12,25]$

$$
I(t)=I_{0} \mathrm{e}^{-t / \tau_{0}}\left[1-2 A_{22}\left(\frac{1}{5}+\frac{4}{5} \cos \Omega t\right) P_{2}(\cos \theta)\right],
$$

where the perturbation function $G_{22}(t)$ is expressed as $G_{22}(t)=I / 5+(4 / 5) \cos \Omega t$, and $\hbar \Omega$ is the quadrupole splitting of the excited state. In case of $\theta=0, P_{2}(\cos \theta)=1$, one gets the strongest SRPAC signal. Figure I2.4 shows theoretical SRPAC spectra with quadrupole splitting in different $\theta$.

12.2.2.2 Magnetic Hyperfine Interactions Next, we will consider the SRPAC intensity in the presence of magnetic hyperfine interactions. In the case of randomly oriented nuclear magnetic moments, $R(t)$ takes the form [18]

$$
R(t)=P_{2}(\cos \theta)\left(1+2 \cos \omega_{\mathrm{B}} t+2 \cos 2 \omega_{\mathrm{B}} t\right) / 5,
$$


where $\omega_{\mathrm{B}}=-g_{\mathrm{N}}^{\mathrm{e}} \mu_{\mathrm{N}} \mathbf{B} / \hbar$ is the Larmor frequency, proportional to the g-factor of the nuclear excited state $g_{N}^{e}$ and to the magnetic field $\mathbf{B}, \mu_{N}$ is the nuclear magneton. In the case of the magnetic moments aligning along the magnetic field $\mathbf{B}$, it can be shown that the anisotropy factor $R(t)$ has the form [I8]

$$
R(t)=C_{0}+C_{1} \cdot \cos \left(\omega_{\mathrm{B}} t-\phi\right)+C_{2} \cdot \cos \left(2 \omega_{\mathrm{B}} t-2 \phi\right),
$$

where $C_{0}, C_{1}$, and $C_{2}$ are

$$
\begin{aligned}
& C_{0}=\left(1+3 \cos 2 \theta_{0}\right)\left(1+3 \cos 2 \theta_{2}\right) / 16 \\
& C_{1}=3 \sin 2 \theta_{0} \sin 2 \theta_{2} / 4 \\
& C_{2}=3\left(1-\cos 2 \theta_{0}\right)\left(1-\cos 2 \theta_{2}\right) / 16
\end{aligned}
$$

The angles $\theta_{0}$ and $\theta_{2}$ are between the direction of the magnetic field $\mathbf{B}_{\text {ext }}$, the direction of the magnetic polarization $\mathbf{B}$ of the incoming SR photon, and the direction of the scattered photon $\mathbf{k}_{\text {out }}$ (Fig. 12.4), $\phi$ is the angle between the projection of $\mathbf{B}$ and $\mathbf{k}_{\text {out }}$ onto the plane perpendicular to $\mathbf{B}_{\text {ext }}$, and it determines the phase of the quantum beats in SRPAC in the case of magnetic hyperfine interactions. The sign of $\phi$ is defined by right-hand rule. The theoretical SRPAC spectra described by 12.7 and 12.8 are shown in Fig. 12.4 .

\subsubsection{Experimental Aspects of NFS and SRPAC}

12.2.3.1 General Aspects of the Experimental Setup Only with the emergence of the third-generation synchrotron radiation sources and the development of advanced X-ray optics and X-ray detection methods, NRS using SR became feasible. At present, there are three major synchrotron radiation facilities for NRS experiments. They are beamline 3ID [27] at the Advanced Photon Source (APS) at Argonne National Laboratory in the USA, beamlines ID I 8 and ID22N [28] at the European Synchrotron Radiation Facility (ESRF) in France, and beamline BL09XU [29] at SPring-8 in Japan. Beamline NE3 at the Photon Factory Accumulate Ring (PF-AR) at the High Energy Accelerator Research Organization (KEK) in Japan, one of the first beamlines to perform NFS experiments, is still under operation. The experimental data shown in this chapter were obtained at beamline NE3 of PF-AR and beamline BL09XU of SPring-8. The schematic view of the experimental setup of NFS and SRPAC is shown in Fig. 12.5 [8, I2,30,3I].

SR Source The average brightness of SR beam, which is a measure of the $X$-ray flux density per unit phase space volume, is the most important quantity for nuclear resonance experiments [8,30]. In the current third-generation synchrotron radiation sources, the maximum brightness of the undulator radiation is $\sim 10^{22}$ photon $\mathrm{s}^{-1}$ ( $\mathrm{mrad}^{2} 0.1 \%$ bandwidth) $[8,30]$. As a comparison, a regular commercially available radioactive Mössbauer source with $100 \mathrm{mCi}$ activity, such as a ${ }^{57} \mathrm{Co}$ source, the brightness is $\sim 10^{13}$ photon $\mathrm{s}^{-1}$ ( $\mathrm{mrad}^{2} 0.1 \%$ bandwidth) at $30 \mathrm{~cm}$ distance. However, the SR beam generated after the undulator (the so-called "white beam") contains a broad energy spectrum ranging from several electron volts up to a few hundred killoelectron volts with distinct peaks of enhanced intensity and energy bandwidths of about a few hundred electron volts. It is not suitable for nuclear resonance experiments because the very strong nonresonant radiation, which is unwanted in those experiments, has to be handled by the detector and the subsequentacquisition electronics. To selectonlya small portion of energy from this broad energy spectrum with narrow energy bandwidth $(\sim$ meV) centered at the nuclear resonance energy (14.4 keV in case of ${ }^{57} \mathrm{Fe}$ experiments), monochromatization is performed.

Monochromators A two-step monochromatization is performed in NRS experiments [8,30,3 I]. In the first step, a high heat load monochromator (HLM) is used to select the nuclear resonance energy with the bandwidth of a few electron volts from the white beam by utilizing the Bragg diffraction condition. It is not enough for suppressing the nonresonant radiation in NFS and SRPAC experiments. However, it reduces the power of the $X$-ray beam to $0.1 \mathrm{~W} \mathrm{~cm}^{-2}, 10^{6}$ times less than the white beam power (several $\mathrm{kW} \mathrm{cm}^{-2}$ ). HLM typically consists of twoSi ( I I I) crystals or diamond (I I I) crystals. Since the first crystal has to stand high heat loads from the write beam, different cooling methods are used. For example, at BL09XU, SPring8, two liquid $\mathrm{N}_{2}$-cooled silicon ( $\mathrm{I} \mathrm{I} \mathrm{I}$ ) crystals are used. At beamline NE3 of the PF-AR, two water-cooled (I I I) diamond crystals are used due to their excellent thermal conductivity, low absorption for X-rays, low-induced vibrations, and the simplicity introduced by avoiding a complicated cooling system.

To further reduce the energy bandwidth of the SR from several electron volts generated by HLM to approximately millielectron volts and provide energy tunability over $\sim 100 \mathrm{meV}$ required for NRS experiments, a high-resolution monochromator (HRM) is used in the second step of monochromatization. To achieve energy bandwidth reduction and 

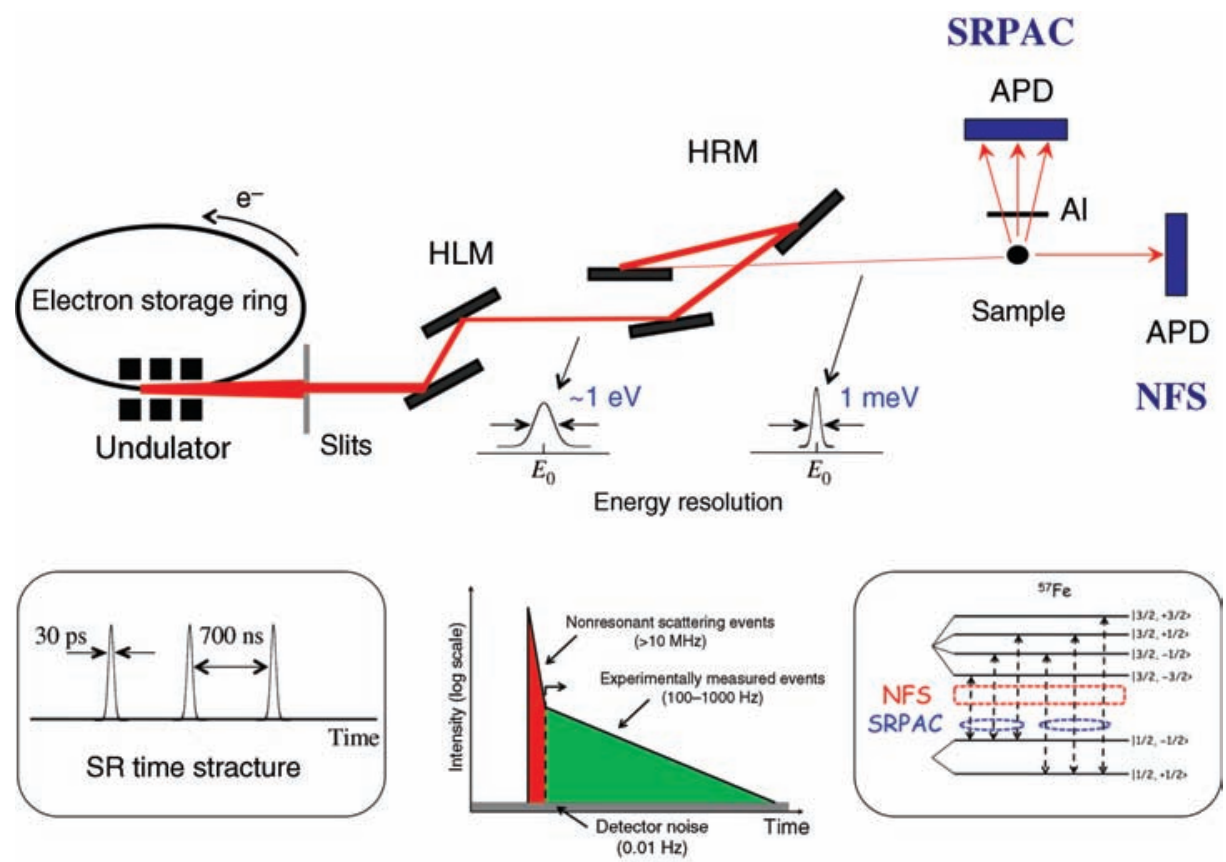

\section{FIGURE 12.5}

Top: Experimental setups for NFS and SRPAC. Bottom: The SR time structure (left), the detection scheme utilizing the time-discrimination trick described in the text (middle), and the scheme describing the allowed nuclear transitions that interfere and give quantum beats observed in NFS and SRPAC.

in the meantime maintain an acceptable transmission of the available spectral flux, unique designs utilizing Bragg diffraction conditions as well as X-ray beam dynamics are achieved. For detailed discussions on HRM, one should refer to Ref. 30 and references therein. The HRM at BL09XU of the SPring-8 uses asymmetrically cut $\operatorname{Ge}(4,2,2)$ and two $\mathrm{Si}(9,7,5)$ crystals. The flux at SPring-8 is $\sim 3 \times 10^{9}$ in a $1.1 \mathrm{meV}$ bandwidth. The HRM at beamline NE3 of the PF-AR uses two $\mathrm{Si}$ channel cuts with $\mathrm{Si}(4,2,2)$ and $\mathrm{Si}(12,2,2)$, it provides $\sim 5 \times 10^{8}$ photons s${ }^{-1}$ in $6.4 \mathrm{meV}$ bandwidth at $14.4 \mathrm{keV}$.

Apart from HRM for ${ }^{57} \mathrm{Fe}$ applications, HRMs for nuclear resonant energies of other Mössbauer isotopes have also been developed to perform NRS experiments, they include ${ }^{181} \mathrm{Ta}(6.124 \mathrm{keV}),{ }^{169} \mathrm{Tm}(8.410 \mathrm{keV}),{ }^{83} \mathrm{Kr}(9.404 \mathrm{keV}),{ }^{151} \mathrm{Eu}$ $(21.514 \mathrm{keV}),{ }^{149} \mathrm{Sm}(22.496 \mathrm{keV}),{ }^{19} \mathrm{Sn}(23.88 \mathrm{keV}),{ }^{161} \mathrm{Dy}(25.65 \mathrm{I} \mathrm{keV}),{ }^{40} \mathrm{~K}(29.83 \mathrm{keV}),{ }^{121} \mathrm{Sb}(37.133 \mathrm{keV})$, and ${ }^{157} \mathrm{Gd}$ $(63.917 \mathrm{keV})[8,30]$.

Detectors and Detection Methods As mentioned in the earlier sections of the theoretical aspects of NFS and SRPAC, the signal analyzed in these experiments is the nuclear decay signal from the nuclear excited states. However, right after the incident SR beam hits the sample, a huge nonresonant scattering signal originated from electron scattering and absorption is generated together with the nuclear decay signal. The total scattered photons received by the detector can be up to $6 \times 10^{6}$ counts $^{-1}$ even with the incident SR beam of approximately millielectron volt energy bandwidth centered at $14.4 \mathrm{keV}$ (in case of ${ }^{57} \mathrm{Fe}$ ). Among these photons, only $<0.1 \%$ is belong to the nuclear decay signal. To remove all the background noise generated by nonresonant scattering signal, the time-discrimination trick based on different timescale of different scattering process and the unique time structure of SR radiation are utilized (Fig. 12.5) [8,30]. The typical timescale of nonresonant scattering is in the order of femtosecond, while nuclear level lifetime is in the order of several to several hundred nanoseconds, the time-discrimination trick can be used to separate the NRS signal from the nonresonant scattering signal. Furthermore, the $S R$ is not continuous, instead, it arrives in discreet pulses with the duration of a pulse in the range of 50-200 ps and the interval between pulses in the range of several to several hundred nanoseconds for typical third-generation synchrotron sources (Fig. 12.5) [8,30]. This discontinuity of SR is called time structure of SR, which makes the time-discrimination trick possible for NRS experiments.

To experimentally achieve the time-discrimination trick, the detectors should be able to sustain an intense pulses of prompt scattering (up to $10^{9}$ photons $\mathrm{s}^{-1}$ ) during the flash of SR and several nanoseconds later to detect nuclear decay signals with usually $<10^{3}$ photons $\mathrm{s}^{-1}$. Only a few detectors can meet these severe requirements. The best performance 
is achieved by avalanche photodiode (APD) detectors [8,30]. The main advantages of APD detectors are their good count-rate capability (up to $\sim 10^{9}$ photons $\mathrm{s}^{-1}$ ), good time resolution $\left(<\mathrm{Ins}\right.$ ), and low noise $\left(\sim 0.0 \mathrm{l}\right.$ counts $\mathrm{s}^{-1}$ ). For recording high-resolution time spectra, such as NFS spectra, an APD detector with small detection area is preferred. While in SRPAC experiments, a large-area APD detector or detector array is preferred to catch more delayed fluorescence. For more detailed discussion of the development in this area, please refer to Refs. 8,30 and references therein. For the experiments present in this chapter, a $2 \times 2$ array APD with four $3 \times 5 \mathrm{~mm}^{2}$ area was used for SRPAC experiments at SPring-8, an eight-stacked APDs in grazing incident geometry with surface area of I $\times 2.5 \mathrm{~mm}{ }^{2}($ A.Q.R. Baron, Y. Tanaka, T. Ishikawa, SPring-8 Research Frontiers, S. Kikuta, ed., 2000/200I, unpublished) were used for NFS experiments at SPring-8, and four-stacked APDs with surface area of $2.8 \mathrm{~mm}$ in diameter were used for NFS experiments at PF-AR [32]. Apart from detectors, a proper setup of time electronics is also needed to block the nonresonant scattering signal (see Fig. 12.5).

To get optimum NRS signal, the pulse separation of SR should also be chosen to be in the order of the lifetime of the resonant nucleus, in case of ${ }^{57} \mathrm{Fe}$, the nuclear level lifetime is $\tau=141.1 \mathrm{~ns}$. For NFS and SRPAC experiments, the pulse separation of SR ideally should be as long as possible, since it determines the spectral resolution. As mentioned in Ref. 33, the energy resolution of NFS is

$$
\delta E=\Gamma \operatorname{coth}\left(\frac{\Gamma \delta t}{4 \hbar}\right)
$$

where $\delta t$ is given by the SR pulse separation minus the detector dead time. For the experiments present in this chapter, a SR pulse separations of $\sim 220$ and $\sim 600$ ns were used at Spring-8, and of $\sim 1000$ ns was used at PF-AR.

12.2.3.2 Differences of the Experimental Setup in NFS and SRPAC In NFS setup (Fig. I2.5), the HRM is fixed at the resonance energy, for ${ }^{57} \mathrm{Fe}$ it is $14.4 \mathrm{keV}$, the APD detector is placed behind the sample and along the incident beam direction. The typical sample-detector distance is $\sim 1 \mathrm{~m}$. Since the NFS signal is a coherent scattering signal, which is well collimated in the forward direction, no loss of the signal due to divergence of the beam occurs. Moreover, by placing the detector far from the sample, any incoherent scattering signal can be completely eliminated. To reduce the nonresonant incident energy, a I.I meV energy resolution HRM is used at SPring-8, and a 6.4 meV energy resolution HRM is used at PF-AR. To obtain higher spectral resolution and higher detector efficiency, eightstacked APDs in grazing incident geometry with surface area of $\mathrm{I} \times 2.5 \mathrm{~mm}^{2}$ (SPring-8) and four-stacked APDs with surface area of $2.8 \mathrm{~mm}$ in diameter (PF-AR) are used. The data presented in this chapter were measured under room temperature and cryogenic temperatures down to $4.2 \mathrm{~K}$. A homemade cryostat was used at SPring-8, and an Oxford Optistat SXM liquid helium bath cryostat was used at PF-AR. A permanent magnet with up to $0.3 \mathrm{~T}$ magnetic field was used at SPring-8, and a superconducting magnetic with magnetic field up to $7 \mathrm{~T}$ was used at PF-AR to magnetize samples.

In SRPAC setup (Fig. 12.5), the HRM is also fixed at resonance energy. As pointed out earlier, SRPAC signal is independent from Lamb-Mössbauer factor $f_{L M}$ by covering all phonon-assisted processes using a large enough energy bandwidth incident radiation, so SRPAC experiments do not require a very high-resolution HRM. However, to avoid detector saturation due to high photon flux from large energy bandwidth incident radiation, a $3.5 \mathrm{meV} \mathrm{HRM} \mathrm{is} \mathrm{used} \mathrm{at}$ SPring-8 for SRPAC experiments. To obtain strongest SRPAC signals, the APD detector is place in $90^{\circ}$ geometry (Fig. 12.5). Since SRPAC signals have angular distribution, and in the real experiments the detectors have finite area, angular averaging effect exists, which can reduce the contract of observed quantum beats. Sergueev [25] discussed the optimum experimental geometry in detail. According to his discussion, a sample-detector distance of $\sim 8 \mathrm{~mm}$ for a $2 \times 2$ array APD detector with four $3 \times 5 \mathrm{~mm}^{2}$ detection area APDs used at SPring-8 can generate an optimum experimental geometry.

When the HRM is fixed at resonance energy in SRPAC experiments, contributions from $4 \pi$ scattering of NFS will be observed in SRPAC. The incoherent nuclear scattering is not the only channel of scattering producing delayed radiation into $4 \pi$ solid angle, also the combination of NFS and subsequent Rayleigh scattering can direct delayed radiation into the full solid angle [34]. This additional contribution can be so strong that it completely overshadows the SRPAC signal. An easy way to eliminate this NFS $4 \pi$ scattering and get pure SRPAC signal is tuning the incident energy out of the resonance by $10-100 \mathrm{meV}$ [35]. Then the resonant absorption occurs with creation or annihilation of phonons, which prohibits nuclear coherent scattering. However, the absorption cross section reduced dramatically when moving away from the resonance. As a result, the SRPAC signal detected away from the resonance is much smaller than the signal detected on the resonance. 
In Fig. 12.5, an aluminum foil is also used in SRPAC experiments placed in between the sample and the detector. SRPAC detects the direct nuclear fluorescence signal. But the nuclear decay can also follow the route where nuclear fluorescence experiences internal conversion and finally generates electronic fluorescence, mainly $\mathrm{K}$ shell fluorescence. The internal conversion coefficient for ${ }^{57} \mathrm{Fe}$ is 8.6 that means only $\sim 1$ out of 10 photons detected for the incoherent scattering is from nuclear fluorescence, which bears SRPAC information. To remove the $\mathrm{K}$ shell fluorescence, an aluminum foil is used. In the case of ${ }^{57} \mathrm{Fe}$, the energy of the nuclear fluorescence is $14.4 \mathrm{keV}$, the $\mathrm{K}$ shell fluorescence mainly is $\mathrm{K} \alpha$ fluorescence, which has the energy of $6.4 \mathrm{keV}$. The attenuation lengths through aluminum foil for these two energies are $I_{6.4} \sim 40 \mu \mathrm{m}$, and $I_{14.4} \sim 440 \mu \mathrm{m}$. So applying $300 \mu \mathrm{m}$ thick aluminum foil reduces $\mathrm{K} \alpha$ fluorescence by $>99.5 \%$, while only reduces nuclear fluorescence by $\sim 50 \%$.

\subsubsection{Sample Requirements}

NFS As discussed in Section 2.I, the dynamic beat generated by a thick sample can be observed in NFS spectra, it will mix with quantum beat, which is generated by hyperfine interactions, to give a complicated NFS spectra. To simplify data analysis, one should prepare a sample with suitable thickness. In practice, a sample with effective thickness $t_{\text {eff }} \leq 20$ is an optimum sample thickness for NFS experiments. In our experiments, the volume of the sample cells is $\sim 25 \mu \mathrm{l}$, so for protein solution samples, ${ }^{57} \mathrm{Fe}$ concentration of $\mathrm{I}-5 \mathrm{mM}$ can generate a sample with a good effective thickness. For powder samples, usually I-2 mg of sample enriched with ${ }^{57} \mathrm{Fe}$ is needed, the sample then is mixed evenly with boron nitride (BN) to fill the space of the sample cells. The typical measurement time is $\sim 24-36 \mathrm{~h}$ for a protein frozen solution sample and $\sim 12-24 \mathrm{~h}$ for a solid powder sample.

SRPAC InSRPAC experiment, the directnuclearfluorescencesignal $\left(14.4 \mathrm{keV} \mathrm{for}{ }^{57} \mathrm{Fe}\right)$ is detected. The attenuation length of I $4.4 \mathrm{keV}$ radiation is $\sim 5 \mathrm{~mm}$ in water. Practically, a I- $2 \mathrm{~mm}$ sample thickness is used. The ${ }^{57} \mathrm{Fe}$ concentration in the samples should be as high as possible. A $>5 \mathrm{mM}$ concentration is preferred for solution samples. Considering the general difficulty of generating high-concentration protein samples, a sample with the minimum ${ }^{57} \mathrm{Fe}$ concentration of $2 \mathrm{mM}$ is needed to get a decent spectrum with acceptable statistics. The typical measurement time is $\sim 16-36 \mathrm{~h}$ for a protein frozen solution sample and $\sim 6-12 \mathrm{~h}$ for a solid powder sample.

\subsection{APPLICATIONS IN BIOINORGANIC CHEMISTRY}

\subsubsection{Nuclear Forward Scattering}

Recently, SR-based NFS has been applied to bioinorganic chemistry to reveal the electronic structure of the iron center in heme model complexes as well as myoglobin proteins, it has also been used to illustrate the paramagnetic relaxation at the iron sites in heme model complexes and dynamic structural disorder of dioxygen $\left(\mathrm{O}_{2}\right)$ binding on the iron site of oxymyoglobin [16] (for the application of NFS to heme proteins, please refer to Chapter 16, to other research areas, please refer to Chapters 10 and 40). These studies showed that NFS is a promising technique for illustrating the electronic structure and dynamics at the iron site of the heme center and related model complexes. With a long detection window, NFS has higher sensitivity on the hyperfine interaction parameters than the conventional Mössbauer spectroscopy; therefore, more accurate simulation parameters can be obtained (see Eq. (I2.9)).

In this section, the application of NFS for the first time to the study of iron-sulfur (FeS) proteins will be presented. FeS proteins host unique metal clusters, called FeS clusters, which are one of the most ancient, versatile and multifunctional prosthetic groups found in biology. They are commonly involved in electron transport, catalysis, and regulatory processes in living cells (for recent reviews, see [36,37]). Studies of FeS clusters in proteins are always an important step toward the understanding of the function of these proteins. Conventional Mössbauer spectroscopy has been used extensively in this area, and achieved tremendous success in elucidating the geometric and electronic structures of these clusters [2,3]. Yet, a search for better spectroscopic techniques is always needed when it comes to the studies of complicated iron-sulfur proteins, such as nitrogenase and hydrogenase, which have 10-30 different iron atoms in each protein molecule. Due to its unique features discussed in Section 12.2, NFS shows great potential to be one of such techniques. In the following, a brief introduction on the FeS proteins included in this section will be presented.

[2Fe-2S] Ferredoxin VI from Rhodobacter capsulatus [2Fe-2S] ferredoxins contain a single $\mathrm{Fe}_{2} \mathrm{~S}_{2}(\mathrm{~S}-\mathrm{cys})_{4}$ center (Fig. 12.6) . Most of the proteins can be catalogued into three main subgroups, namely, plant-type, mammalian-, 


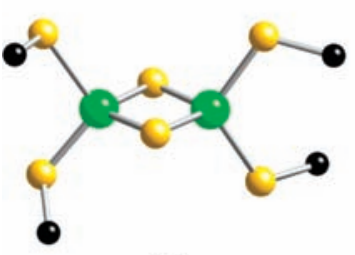

(a)

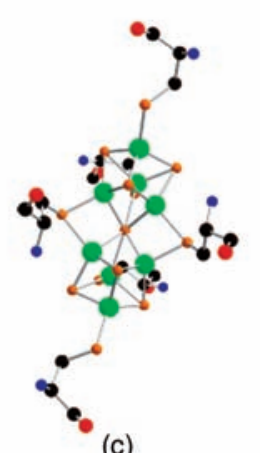

(c)

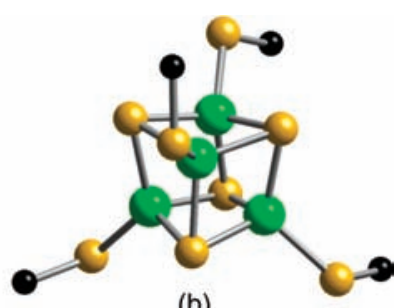

(b)

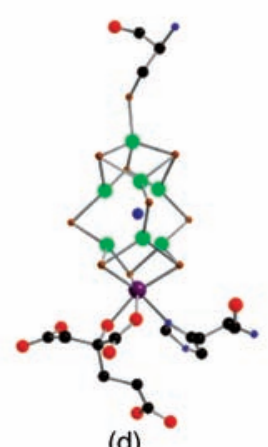

FIGURE 12.6

Molecular structure of (a) [2Fe2S] cluster of ferredoxin $\mathrm{VI}$ from $R$. capsulatus (PDB: 1E9M), (b) [4Fe4S] cluster of ferredoxin D14C from $P$. furiosus (PDB: 2Z8Q), (c) P-cluster of Mo-nitrogenase from $A$. vinelandii (PDB: $1 \mathrm{M} 1 \mathrm{~N})$, and (d) FeMo-co of Monitrogenase from $A$. vinelandii (PDB: 1M1N). Color scheme: Fe (green), Mo (purple), S (yellow), C (black), $\mathrm{O}$ (red), and the interstitial atom $X$ (blue).

vertebrate-, or hydroxylase-type, and Isc-type ferredoxins. In the first two subgroups, [2Fe-2S] ferredoxins function as electron transfer proteins [38-40]. In the third subgroup, ferredoxins play a role in Fe-S cluster biosynthesis [4I]. R. capsulatus is a photosynthetic bacterium with six distinct ferredoxin-encoding genes [42], and the sixth type- $\mathrm{Rc} F d \mathrm{VI}-\mathrm{is}$ involved in FeS cluster biosynthesis [43]. The crystal structure of Rc FdVI revealed a similar Fe center comparing with other ferredoxins [43]. Although Mössbauer data of Rc FdVI has not been reported, ferredoxins of other types in different bacteria have been studied extensively using Mössbauer spectroscopy [37,44-48]. In oxidized form, ferredoxins contain a $\mathrm{Fe}(\mathrm{III}) \mathrm{Fe}(\mathrm{III})$ diamagnetic center $\left(S_{\text {tot }}=0\right)$. In the reduced form, a Fe(II)Fe(III) dimer with $S_{\text {tot }}=\mathrm{I} / 2$ is present. The oxidized ferredoxin Mössbauer spectrum only showed a quadrupole doublet with an averaged quadrupole splitting $\Delta E_{\mathrm{Q}} \sim 0.6-0.7 \mathrm{~mm} \mathrm{~s}^{-1}$, isomer shift $\delta \sim 0.3 \mathrm{~mm} \mathrm{~s}^{-1}$ even down to liquid helium temperature $(4.2 \mathrm{~K})$, which confirms the diamagnetism of the two iron center. This diamagnetism comes from the spin exchange coupling of the two irons [37]. In the reduced form, two quadrupole doublets with different isomer shifts $\left(\sim 0.3 \mathrm{~mm} \mathrm{~s}^{-1}\right.$ versus $\left.\sim 0.6 \mathrm{~mm} \mathrm{~s}^{-1}\right)$ and quadrupole splittings $\left(\sim 0.6 \mathrm{~mm} \mathrm{~s}^{-1}\right.$ versus $\left.\sim 3 \mathrm{~mm} \mathrm{~s}^{-1}\right)$ can be observed, which is consistent with the presence of both $\mathrm{Fe}^{2+}$ and $\mathrm{Fe}^{3+}$ in the protein. Under liquid helium temperature and external magnetic field, magnetic sixtet are shown, different magnetic properties are observed for $\mathrm{Fe}^{3+}$ and $\mathrm{Fe}^{2+}$ center. In this section, oxidized Rc FdVI is examined by ${ }^{57} \mathrm{Fe}$ NFS.

[4Fe-4S] Ferredoxin from Pyrococcus furiosus [4Fe-4S] ferredoxins are electron transfer agents. In general, they consist of a $\mathrm{Fe}_{4} \mathrm{~S}_{4}$ cluster with four irons terminally linked with four cysteine ligands to the protein scaffold (Fig. 12.6). However, in [4Fe-4S] ferredoxin from $P$. furiosus (Pf), the 4Fe cluster is coordinated by three cysteine ligands (CII, CI 7, and $\mathrm{C56}$ ) and one aspartate ligand (DI4). Thanks to its small size and high stability at even $95^{\circ} \mathrm{C}$, as well as the ready interconversion between [4Fe-4S] and [3Fe-4S] clusters, the DI4 site of the PfFd can be mutated to a cysteine ligand to form PfD I4C Fd mutant [49-5I]. In this chapter, the oxidized form of this mutant is examined by ${ }^{57}$ Fe NFS. Mössbauer spectra of [4Fe-4S] ferredoxin from Bacillus stearothermophilus [52] and Clostridium pasteurianum [53,54] have been reported previously. In oxidized form, the protein contains a $[4 \mathrm{Fe}-4 \mathrm{~S}]^{2+}$ cluster with two electron delocalized $\mathrm{Fe}^{2.5+} \mathrm{Fe}^{2.5+}$ pairs, the spins are antiparallel paired to yield a diamagnetic center $\left(S_{\text {tot }}=0\right)$. In the low external magnetic field, the oxidized [4Fe-4S] clusters show a quadrupole doublet down to $4.2 \mathrm{~K}$ with the averaged isomer shift $\sim 0.45 \mathrm{~mm} \mathrm{~s}^{-1}$, and the averaged quadrupole splitting $\sim 1.0 \mathrm{~mm} \mathrm{~s}^{-1}$.

Nitrogenase Nitrogenase is an important iron-sulfur protein, which catalyzes the ATP-dependent reduction of dinitrogen $\left(\mathrm{N}_{2}\right)$ into ammonia $\left(\mathrm{NH}_{3}\right)$. This reaction, termed biological nitrogen fixation, represents a major entry point of nitrogen into the global nitrogen cycle. There are three types of nitrogenases, molybdenum-, vanadium-, and iron-only nitrogenase. Among them, Mo-nitrogenase is responsible for the majority of biological fixation activity, and is most 
commonly studied [55]. Mo-nitrogenase utilizes two of the most complex FeS clusters, P-cluster and iron-molybdenum cofactor (FeMo-co) (Fig. 12.6), to break the strong $\mathrm{N}_{2}$ triple bond under ambient conditions. P-cluster and FeMo-co are embedded in MoFe protein, one of the two component proteins in Mo-nitrogenase. P-cluster is a unique [8Fe-7S] cluster, which most likely functions as electron relay unit, accepting electrons from the [4Fe-4S] cluster in Fe protein, the other component protein in Mo-nitrogenase, and donating them to FeMo-co, where $\mathrm{N}_{2}$ reduction is completed. FeMo-co, the active center for $\mathrm{N}_{2}$ reduction, is composed of a unique Mo-7Fe-9S-X cage-like cluster and an $R$-homocitrate that is coordinated at Mo. $X$ is an interstitial light atom sitting in the center of the cage, its identity is still unknown, and most likely is $\mathrm{C}, \mathrm{N}$, or $\mathrm{O}$. In this section, the oxidized Fe protein and the as-isolated MoFe protein are examined by ${ }^{57} \mathrm{Fe}$ NFS. The oxidized Fe protein hosts an [4Fe-4S] ${ }^{2+}$ cluster, which gives a single quadrupole doublets in the low field Mössbauer spectrum down to $4.2 \mathrm{~K}$, which can be fit with the isomer shift of $0.41 \mathrm{~mm} \mathrm{~s}^{-1}$, and the quadrupole splitting of $0.7 \mathrm{I}$ and $1.07 \mathrm{~mm} \mathrm{~s}^{-1}$ [56], resembling other oxidized [4Fe-4S] ferredoxin having a diamagnetic electronic ground state. In the resting state MoFe protein, $\mathrm{P}$-cluster is in the $\mathrm{P}^{\mathrm{N}}$ state having an $\mathrm{S}=0$ electronic ground-state configuration with all $\mathrm{Fe}$ in $\mathrm{Fe}^{2+}$ oxidation state as shown by Mössbauer spectroscopy [57]. The Mössbauer spectrum of the $\mathrm{P}^{\mathrm{N}}$ state $\mathrm{P}$-cluster can be simulated as four quadrupole doublets with an averaged isomer shift of $0.62 \mathrm{~mm} \mathrm{~s}^{-1}$ and quadrupole splittings of 3.00 , $1.34,0.90$, and $0.73 \mathrm{~mm} \mathrm{~s}^{-1}$ at $93 \mathrm{~K}$ [57]. The FeMo-co in the resting state MoFe protein is in the $\mathrm{M}^{\mathrm{N}}$ state having an $\mathrm{S}=3 / 2$ electronic ground-state configuration and a well-characterized EPR signal $(g=4.3,3.7,2.0)[58,59]$. The oxidation states of seven iron atoms in the resting state FeMo-co is most likely to be assigned as $\left[3 \mathrm{Fe}^{3+}, 4 \mathrm{Fe}^{2+}\right]$ as suggested by Mössbauer studies [60]. The zero-field Mössbauer spectrum of the $M^{N}$ state FeMo-co shows a quadrupole doublet with average isomer shift of $0.36 \mathrm{~mm} \mathrm{~s}^{-1}$ and average quadrupole splitting of $0.68 \mathrm{~mm} \mathrm{~s}^{-1}$ at I50 $[60]$.

12.3.1.1 ${ }^{57} \mathrm{Fe}$ NFS of Oxidized Rc FdVI The oxidized Rc FdVI NFS spectra measured at $4.2 \mathrm{~K}$ with and without external magnetic field are shown in Fig. 12.7. The zero-field $4.2 \mathrm{~K}$ spectrum shows a simple beating pattern up to $300 \mathrm{~ns}$ with slow oscillation frequency. The spectrum can be simulated by introducing two iron sites as inspired by the Mössbauer simulations on other types of [2Fe-2S] ferredoxins, the isomer shift of one iron site was fixed at 0 , and used as a reference for another iron site. The best fit gave 0.58 and $0.73 \mathrm{~mm} \mathrm{~s}^{-1}$ for the quadrupole splitting, the isomer shift difference for these two sites was $0.01 \mathrm{~mm} \mathrm{~s}^{-1}$ (Table 12.I). This result was consistent with the result obtained by simulating the zero-field Mössbauer spectrum of the same oxidized Rc FdVI measured at $80 \mathrm{~K}$ (the inset of Fig. 12.7a). Here, two quadrupole splittings of 0.52 and $0.76 \mathrm{~mm} \mathrm{~s}^{-1}$ were obtained with the isomer shifts of 0.27 and $0.28 \mathrm{~mm} \mathrm{~s}^{-1}$. After applying a $7 \mathrm{~T}$ external magnetic field along the polarization direction of the incident beam (Fig. 12.7b), the spectrum shows a more complicated beating pattern with faster oscillation frequencies. The simulation with the internal magnetic field fixed at zero readily reproduced the spectrum well, indicating the observed iron species is diamagnetic. The sign of the quadrupole splitting and the asymmetry parameter $\eta$ can be determined from this magnetic spectrum. From the simulation, we obtained that $\eta=0.5$ and $\Delta E_{Q}>0$ for both iron sites.

\section{FIGURE 12.7}

${ }^{57}$ Fe NFS spectra of oxidized $R c$ FdVI (black circles) measured at $4.2 \mathrm{~K}$ with zero applied magnetic field (top) and with 7.0 T magnetic field applied parallel to the electric field of the incident $X$ rays (bottom). The inset of the top panel shows the zero-field conventional Mössbauer spectrum of the same sample measured at $80 \mathrm{~K}$. The gray curves are the spectral simulations using the parameters presented in the text.

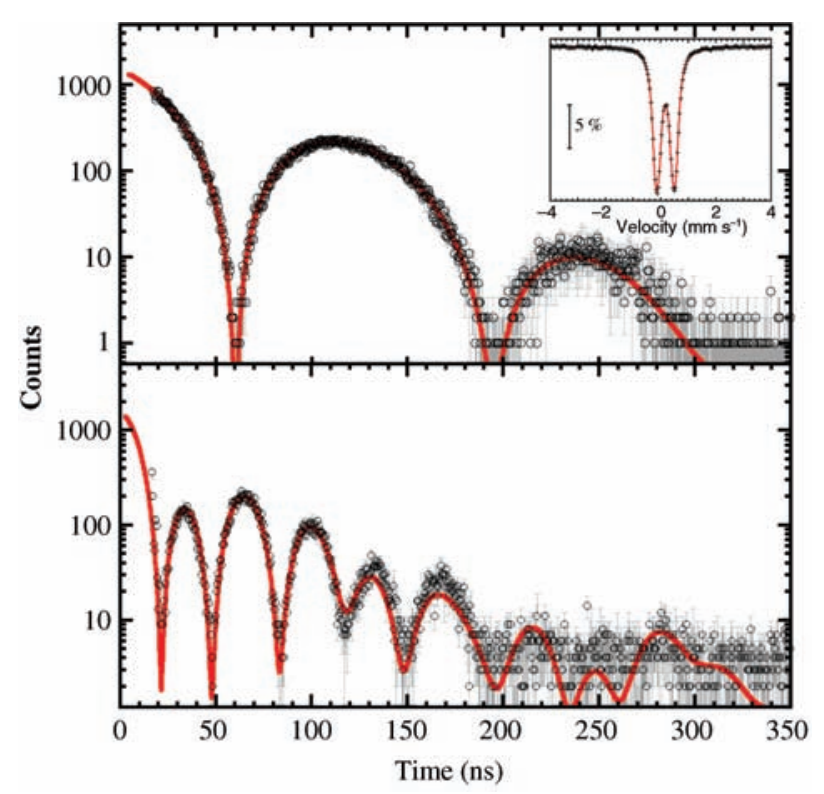


TABLE 12.1 Hyperfine Interaction Parameters Obtained from Zero-Field ${ }^{57} \mathrm{Fe}$ NFS Spectra Compared with the Conventional ${ }^{57} \mathrm{Fe}$ Mössbauer Parameters

\begin{tabular}{|c|c|c|c|c|c|c|}
\hline & \multicolumn{3}{|c|}{ NFS } & \multicolumn{3}{|c|}{ Mössbauer } \\
\hline & $\delta^{a}\left(\mathrm{~mm} \mathrm{~s}^{-1}\right)$ & $\Delta E_{\mathrm{Q}}\left(\mathrm{mm} \mathrm{s}^{-1}\right)$ & $\%^{b}$ & $\delta^{a}\left(\mathrm{~mm} \mathrm{~s}^{-1}\right)$ & $\Delta E_{\mathrm{Q}}\left(\mathrm{mm} \mathrm{s}^{-1}\right)$ & $\%^{b}$ \\
\hline \multirow[t]{4}{*}{ Oxidized Rc FdVI } & $-0.014(3)$ & $0.566(4)$ & 53 & $0.272(7)^{c}$ & $0.52(I)^{c}$ & $50^{c}$ \\
\hline & & & & $0.274(8)^{c}$ & $0.75(I)^{c}$ & $50^{c}$ \\
\hline & & $0.728(7)$ & 47 & $0.26^{d}$ & $0.62^{d}$ & $50^{d}$ \\
\hline & & & & $0.28^{d}$ & $0.76^{d}$ & $50^{d}$ \\
\hline \multirow[t]{8}{*}{ Oxidized PfDI4C Fd } & - & $1.475(2)$ & 25 & $0.42(I)^{c}$ & $1.40(1)^{c}$ & $25^{c}$ \\
\hline & & & & $0.43(1)^{c}$ & $1.13(7)^{c}$ & $25^{c}$ \\
\hline & $-0.007(2)$ & $1.289(3)$ & 25 & $0.42(1)^{c}$ & $0.84(I)^{c}$ & $25^{c}$ \\
\hline & & & & $0.4 I(I)^{c}$ & $0.54(1)^{c}$ & $25^{c}$ \\
\hline & $0.012(2)$ & $1.099(3)$ & 25 & $0.42(1)^{e}$ & $1.50(6)^{e}$ & $25^{\mathrm{e}}$ \\
\hline & & & & $0.43(2)^{e}$ & $1.20(3)^{\mathrm{e}}$ & $25^{\mathrm{e}}$ \\
\hline & $-0.042(2)$ & $0.634(2)$ & 25 & $0.42(2)^{e}$ & $1.10(9)^{\mathrm{e}}$ & $25^{\mathrm{e}}$ \\
\hline & & & & $0.42(2)^{e}$ & $0.66(1)^{e}$ & $25^{\mathrm{e}}$ \\
\hline \multirow[t]{4}{*}{ Oxidized Fe protein } & - & I.I50(2) & 85 & $0.44(1)^{c}$ & $1.23(2)^{c}$ & $60^{c}$ \\
\hline & & & & $0.43(4)^{c}$ & $0.89(4)^{c}$ & $40^{c}$ \\
\hline & $-0.02(2)$ & $0.87(I)$ & 15 & $0.45^{f}$ & $1.22^{f}$ & $75^{f}$ \\
\hline & & & & $0.44^{f}$ & $0.83^{f}$ & $25^{f}$ \\
\hline \multirow[t]{4}{*}{ MoFe protein } & $0.28(I)$ & $2.82(2)$ & 13 & $0.69(2)^{g}$ & $3.02(2)^{g}$ & $13^{g}$ \\
\hline & $0.209(7)$ & $0.77(2)$ & 42 & $0.64(2)^{g}$ & $0.81(2)^{g}$ & $42^{g}$ \\
\hline & $0.18(3)$ & $1.28(6)$ & 5 & $0.64(4)^{g}$ & $1.37(4)^{g}$ & $5^{g}$ \\
\hline & - & $0.72(1)$ & 40 & $0.40(3)^{g}$ & $0.76(3)^{g}$ & $40^{g}$ \\
\hline
\end{tabular}

The numbers in parentheses give the error in the last significant digit.

${ }^{a}$ The isomer shift values in NFS are quoted with respect to one of the iron sites in the simulation. The isomer shift values in Mössbauer data are quoted with respect to $\alpha-\mathrm{Fe}$ foil at $300 \mathrm{~K}$.

${ }^{b}$ The contribution of each Fe site in the simulations.

'Mössbauer data from this work measured at $80 \mathrm{~K}$.

${ }^{d}$ Ref. 45.

${ }^{\mathrm{e}}$ Ref. 52.

Ref. 56.

${ }^{g}$ Refs 59,62.

The NFS spectra of oxidized Rc FdVI revealed a diamagnetic [2Fe-2S] center with an averaged $\Delta E_{\mathrm{Q}}$ of $\sim+0.64$ $\mathrm{mm} \mathrm{s}^{-1}$ and $\eta=0.5$, which is similar with the Mössbauer parameters reported on several other oxidized [2Fe-2S] ferridoxins [44-48], indicating the [2Fe-2S] center in the oxidized Rc FdVI has very similar structural and electronic environments with the $[2 \mathrm{Fe}-2 \mathrm{~S}]$ center in other types of oxidized [2Fe-2S] ferridoxins.

12.3.1.2 ${ }^{57} \mathrm{Fe}$ NFS of Oxidized Pf D14C Fd The oxidized Pf DI4C Fd NFS spectra measured at 4.2 K with and without external magnetic field are shown in Fig. 12.8. The oscillation pattern in the zero-field spectrum is more complicated than the pattern observed in the zero-field spectrum of oxidized Rc FdVI, which indicates more different iron sites may contribute to this spectrum. Same as an oxidized $[2 \mathrm{Fe}-2 \mathrm{~S}]$ center, the $[4 \mathrm{Fe}-4 \mathrm{~S}]^{2+}$ center in oxidized PfDI4C Fd is also diamagnetic. So the oscillation observed in the zero-field spectrum is again generated by the quadrupole splittings. After applying a 7 T magnetic field along the polarization direction of the incident radiation, the external field-induced magnetic splitting is observed, forming an oscillation pattern with faster frequency in the NFS spectrum.

The best fit of the zero-field spectrum was obtained by introducing four different iron sites. The obtained parameters are listed in Table 12.1. Since no reference sample was used, the absolute isomer shift information cannot be obtained. However, the relative isomer shift of each iron site can still be obtained by setting one of the four sites as a reference. The best fit gave four quadrupole splittings as $1.48,1.29,1.1$, and $0.63 \mathrm{~mm} \mathrm{~s}^{-1}$. The relative isomer shift of the last three sites relative to the first site were $-0.007,0.012$, and $-0.042 \mathrm{~mm} \mathrm{~s}^{-1}$, indicating they have very similar isomer shifts. These values are consistent with the zero-field $80 \mathrm{~K}$ Mössbauer measurement on the same sample (the inset of Fig. 12.8a), where the four quadrupole splittings were $1.40,1.13,0.84$, and $0.54 \mathrm{~mm} \mathrm{~s}^{-1}$, the corresponding isomer shifts were 0.42 , 


\section{FIGURE 12.8}

${ }^{57}$ Fe NFS spectra of oxidized $P f$ D14C Fd (black circles) measured at $4.2 \mathrm{~K}$ with zero applied magnetic field (top) and with 7.0 T magnetic field applied parallel to the electric field of the incident $\mathrm{X}$-rays (bottom). The inset of the top panel shows the zero-field conventional Mössbauer spectrum of the same sample measured at $80 \mathrm{~K}$. The gray curves are the spectral simulations using the parameters presented in the text.

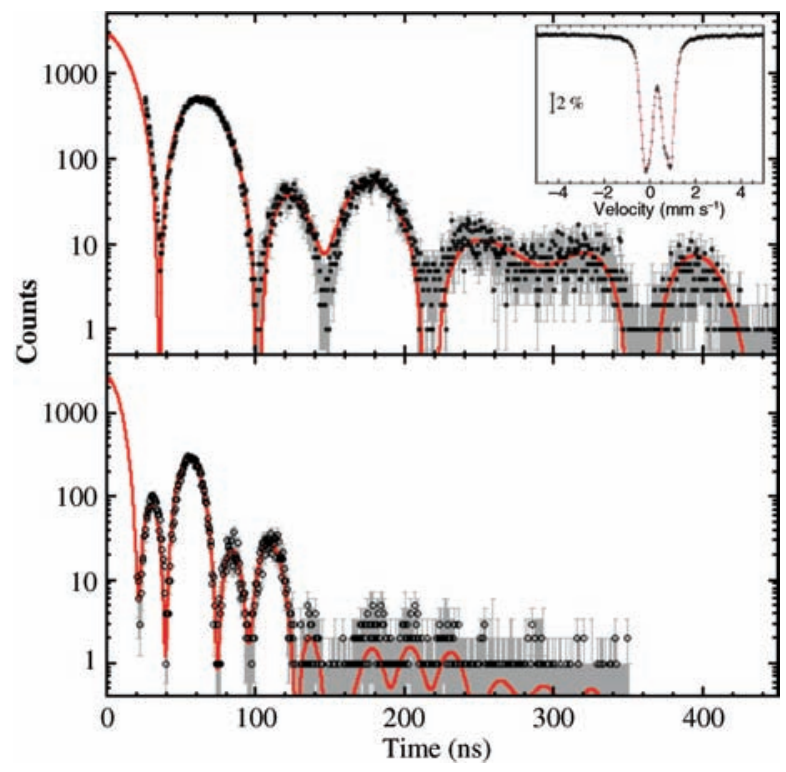

$0.42,0.42$, and $0.46 \mathrm{~mm} \mathrm{~s}^{-1}$. From the $7 \mathrm{~T}$ spectrum, we obtained that for all four iron sites, $\Delta E_{\mathrm{Q}}>0, \eta=0.5$ for two Fe sites, and $\eta=0.7$ for the other two Fe sites.

Both NFS and Mössbauer results on oxidized PfDI4C Fd are very similar with the Mössbauer data on oxidized 4Fe Fd from B. stearothermophilus [52]. Here, four iron sites were used to fit the Mössbauer data. The obtained quadrupole splittings at $4.2 \mathrm{~K}$ were $+1.50,+1.20,+1.10$, and $+0.66 \mathrm{~mm} \mathrm{~s}^{-1}$. The corresponding isomer shifts were $0.42,0.43,0.42$, and $0.42 \mathrm{~mm} \mathrm{~s}^{-1}$. The asymmetry parameter $\eta$ of the first two sites was 0.7 , and of the second two sites was 0.9 . The similarity of the hyperfine interaction parameters of these two oxidize ferredoxins indicates that the $[4 \mathrm{Fe}-4 \mathrm{~S}]^{2+}$ clusters in these two proteins are very similar in structural and electronic environments.

12.3.1.3 ${ }^{57} \mathrm{Fe}$ NFS on Oxidized Fe Protein The zero-field NFS spectrum of the oxidized Fe protein from Azotobacter vinelandii measured at $4.2 \mathrm{~K}$ is present in Fig. 12.9a. Although, the oxidized Fe protein also contains a $[4 \mathrm{Fe}-4 \mathrm{~S}]^{2+}$ cluster, same as the oxidized PfD I4C Fd, they show different beating patterns in the zero-field NFS spectra. For the oxidized Fe protein NFS, the beating pattern is simpler than the oxidized PfDI4C Fd NFS. Instead of introducing four individual Fe sites into the simulation, the oxidized Fe protein NFS spectrum can be well simulated by only introducing two different Fe sites (Table I2.I). The obtained quadrupole splittings of these two Fe sites were I.I5 and $0.87 \mathrm{~mm} \mathrm{~s}^{-1}$ with the relative isomer shift of these two sites as $0.01 \mathrm{~mm} \mathrm{~s}^{-1}$ (Table I2.1). This result was consistent with the result obtained from the Mössbauer spectrum of the same sample measured at $80 \mathrm{~K}$ (the inset of Fig. 12.9a). The Mössbauer spectrum gave two Fe sites with $\Delta E_{\mathrm{Q} 1}=1.24 \mathrm{~mm} \mathrm{~s}^{-1}, \delta_{1}=0.44 \mathrm{~mm} \mathrm{~s}^{-1}$, and $\Delta E_{\mathrm{Q} 2}=0.89 \mathrm{~mm} \mathrm{~s}^{-1}$, $\delta_{2}=0.44 \mathrm{~mm} \mathrm{~s}^{-1}$. The NFS result was also consistent with the published zero-field Mössbauer spectrum of an oxidized Fe protein in [56].

The oxidized PfDI4C Fd and the oxidized Fe protein give similar zero-field Mössbauer spectra, showing a single doublet with an averaged quadrupole splitting of $\sim 1.05 \mathrm{~mm} \mathrm{~s}^{-1}$. However, in NFS spectra, they show quite different beating patterns. This difference directly results in the different number of iron sites used in the simulations. In the oxidized Pf DI4C Fd NFS simulation, four iron sites with different quadrupole splittings were needed, while in the oxidized Fe protein simulation, only two iron sites were needed. In the energy-domain conventional Mössbauer spectroscopy, iron sites with almost the same isomer shift and slightly different quadrupole splittings will only result in a broad quadrupole doublet with unresolved features. However, in time-domain NFS spectroscopy, the same condition will result in a more complicated oscillation pattern of the NFS signal due to the superposition of the slightly different frequencies generated by different iron sites. This shows that NFS has higher sensitivity on the hyperfine interactions parameters than the conventional Mössbauer spectroscopy, given that time window in NFS is long enough (in our case, the NFS single was recorded up to $400 \mathrm{~ns}$, according to Eq. (12.9), the resolution of the NFS spectra of the oxidized PfDI4C Fd and the oxidized Fe protein was much better than $0.2 \mathrm{~mm} / \mathrm{s}$, the best spectral resolution that conventional Mössbauer spectroscopy can reach.). 

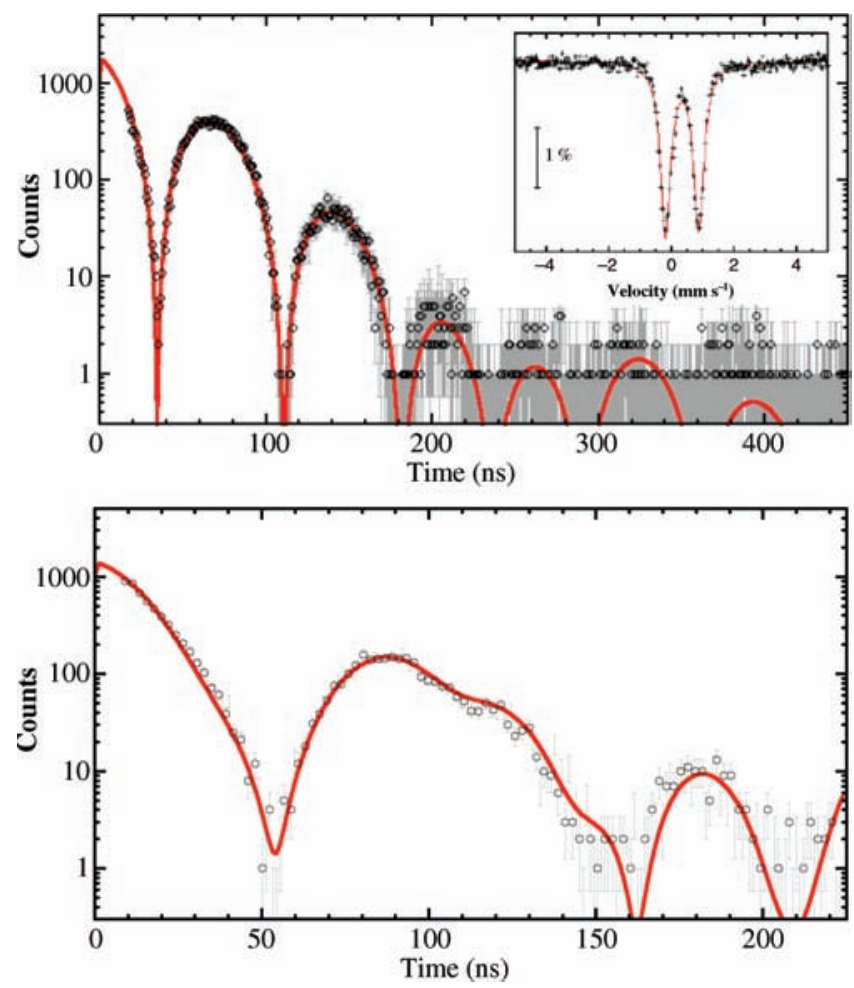

FIGURE 12.9

Zero-field ${ }^{57}$ Fe NFS spectra (black circles) of oxidized Fe protein from $A$. vinelandii at $4.2 \mathrm{~K}$ (top), and the resting state MoFe protein from $A$. vinelandii at $120 \mathrm{~K}$ (bottom). The gray curves are the spectral simulations using the parameters described in the text. The inset of the top panel shows the zero-field conventional Mössbauer spectrum of the oxidized Fe protein sample measured at $80 \mathrm{~K}$.

12.3.1.4 ${ }^{57} \mathrm{Fe}$ NFS on the Resting State MoFe Protein Figure 12.9b shows the zero-field ${ }^{57} \mathrm{Fe}$ NFS spectrum of MoFe protein from A. vinelandii in the resting state recorded up to $220 \mathrm{~ns}$ measured at $150 \mathrm{~K}$. The observed oscillation pattern looks quite simple. Two complete beats were observed, one is observed between 50 and $150 \mathrm{~ns}$, another is observed between 150 and $200 \mathrm{~ns}$. At the first look, this oscillation pattern seems to be similar with the oscillation pattern observed in the oxidized Rc FdVI in Fig. 12.7a. However, different from the oscillation pattern in the oxidized Rc $\mathrm{FdVI}$, where two complete beats have similar oscillation frequency, the two beats in the MoFe protein zero-field NFS have very different oscillation frequencies, also the first beat between 50 and 150 ns actually splits into two beats. This oscillation pattern has not been observed in the FeS protein NFS spectra discussed above. As shown in the previously reported resting state MoFe protein Mössbauer spectra from C. pasteurianum [6I] and A. vinelandii [59,62], four different iron sites were needed to simulate the zero-field spectrum. So we also introduced four iron sites into the NFS simulation while keeping the contribution of each site as the same in the previously reported resting state MoFe protein Mössbauer spectrum from $A$. vinelandii $[59,62]$. The simulation gave $\Delta E_{\mathrm{Q} I}=2.82 \mathrm{~mm} \mathrm{~s}^{-1}, \Delta E_{\mathrm{Q} 2}=0.77 \mathrm{~mm} \mathrm{~s}^{-1}, \Delta E_{\mathrm{Q} 3}=1.38 \mathrm{~mm} \mathrm{~s}^{-1}$, and $\Delta \mathrm{E}_{\mathrm{Q} 4}=0.73 \mathrm{~mm} \mathrm{~s}^{-1}$. Since no reference sample was used during the measurement, the absolute isomer shift values cannot be obtained. However, we can use one of the four sites as a reference to obtain the relative isomer shift values of the other three sites to this reference site. In the simulation, we used the fourth site as the reference, and then we obtained $\delta_{14}=0.28 \mathrm{~mm} \mathrm{~s}^{-1}, \delta_{24}=0.21 \mathrm{~mm} \mathrm{~s}^{-1}$, and $\delta_{34}=0.18 \mathrm{~mm} \mathrm{~s}^{-1}$. Compared with the reported Mössbauer spectra on the resting state MoFe protein from A. vinelandii (Table 12.1) [59,62], the zero-field NFS result is consistent with the zero-field Mössbauer result.

Here we demonstrated the application of ${ }^{57} \mathrm{Fe} \mathrm{NFS}$ to the FeS cluster study for the first time, it showed that NFS technique is capable of obtaining hyperfine interaction parameters commonly encountered in FeS protein studies. Due to the substitution of radioactive source with the high brightness third-generation synchrotron radiation-a much better light source than radioactive sources, NFS spectroscopy is able to achieve better spectral resolution than the conventional Mössbauer spectroscopy, which means more accurate hyperfine interaction parameters can be obtained. This is particularly useful for studying complicated FeS clusters. However, to fully access this feature, long time window (>300 ns) is needed. With the similar ${ }^{57} \mathrm{Fe}$ concentration in samples, the optimal protein sample volume used in NFS experiment can be as small as $20 \mu \mathrm{l}$ due to the small SR beam size, which is 10 times smaller than the optimal sample volume used in conventional Mössbauer spectroscopy. This will be very helpful in some cases where purified proteins cannot be obtained in large volume. 
In this section, we present the ${ }^{57} \mathrm{Fe}$ NFS study mainly on FeS clusters with the diamagnetic electronic ground-state configuration. The use of ${ }^{57} \mathrm{Fe}$ NFS to study FeS clusters in paramagnetic states still needs to be explored. However, in those cases, a spectral simulation program incorporating with spin Hamiltonian formalism [I] is needed. Currently, three NFS simulation programs exist, they are CONUSS [63], which was used in simulating all the spectra presented in this section, SYNFOS [2I], and MOTIF [64]. Among them, only SYNFOS incorporates spin Hamiltonian formalism, and CONUSS has the best user interface. The continuing development of simulation program is crucial for the successful application of NFS technique onto Fe-S protein studies as well as other iron containing metalloproteins in the future.

\subsubsection{SRPAC}

In this section, the application of SRPAC to bioinorganic chemistry is presented. Two model complexes are examined by ${ }^{57} \mathrm{Fe}$ SRPAC, they are an [FeFe] hydrogenase active center model complex, $\mathrm{Fe}_{2}\left(\mathrm{~S}_{2} \mathrm{C}_{3} \mathrm{H}_{6}\right)(\mathrm{CO})_{6}$, and a ferrimagnetic compound, $\mathrm{Ni}$ ferrite $\left(\mathrm{NiO} \cdot \mathrm{Fe}_{2} \mathrm{O}_{3}\right)$. For the former complex, ${ }^{57} \mathrm{Fe}$ SRPAC was used to reveal the electric quadrupole splittings at the iron center, and for the latter one, ${ }^{57} \mathrm{Fe} \mathrm{SRPAC}$ was used to probe the internal magnetic field at the iron center. In addition, ${ }^{61} \mathrm{Ni}$ SRPAC has also been applied to Ni ferrite to probe the internal magnetic field at the Ni center. The SRPAC-derived hyperfine interaction parameters at both the Fe site and the Ni site of these two complexes are consistent with the parameters obtained by conventional Mössbauer spectroscopy, indicating that SRPAC is a promising new technique to study hyperfine interactions of Mössbauer isotopes in bioinorganic chemistry.

12.3.2.1 ${ }^{57} \mathrm{Fe}$ SRPAC of $\mathrm{Fe}_{2}\left(\mathrm{~S}_{2} \mathrm{C}_{3} \mathrm{H}_{6}\right)(\mathrm{CO})_{6} \mathrm{Fe}_{2}\left(\mathrm{~S}_{2} \mathrm{C}_{3} \mathrm{H}_{6}\right)(\mathrm{CO})_{6}$ is a dinuclear complex [65], mimicking FeFe hydrogenase active center- $\mathrm{H}$ cluster $2 \mathrm{Fe}$ center ([66] and references therein). Each of the two $\mathrm{Fe}(\mathrm{l})$ centers is terminally ligated with three $\mathrm{CO}$ ligands, a bridging thiolate ligand- $\left[\mathrm{SCH}_{2} \mathrm{CH}_{2} \mathrm{CH}_{2} \mathrm{~S}\right]^{2-}$ (the inset of Fig. 12.10a). Previous Mössbauer studies on this complex [67] revealed that the two $\mathrm{Fe}(\mathrm{I})$ centers are inequivalent, giving two quadrupole doublets with isomer shifts of 0.03 and $0.04 \mathrm{~mm} \mathrm{~s}^{-1}$, quadrupole splittings of 0.70 and $0.87 \mathrm{~mm} \mathrm{~s}^{-1}$, respectively.

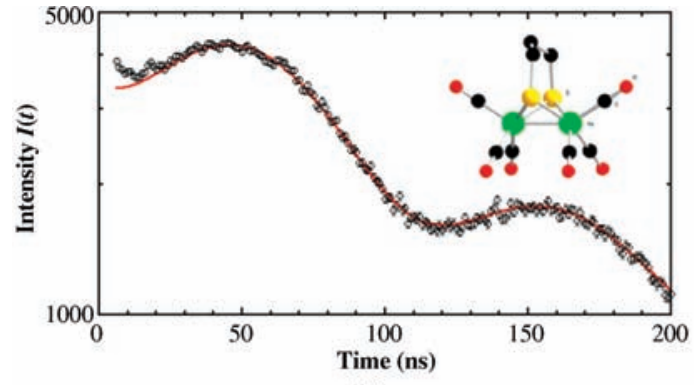

(a)

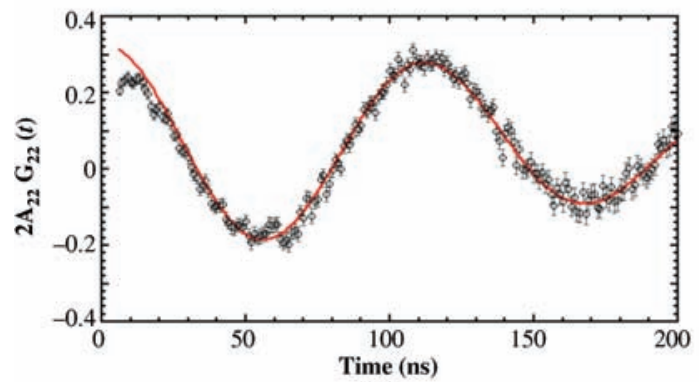

(c)

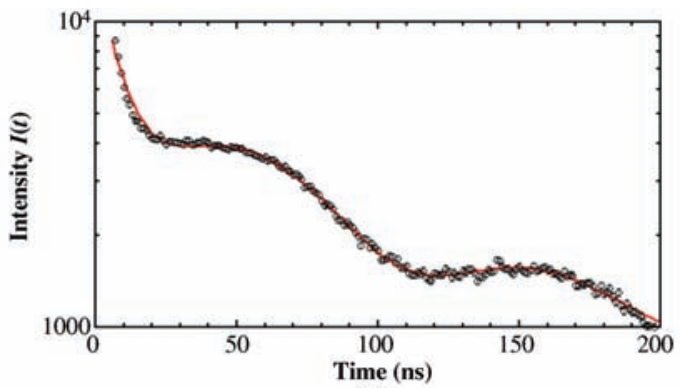

(b)

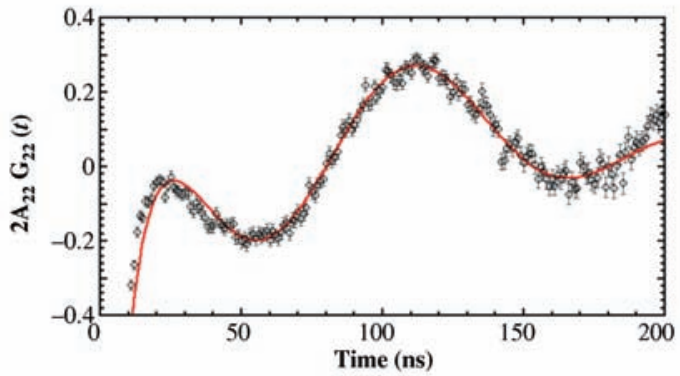

(d)

FIGURE 12.10

${ }^{57} \mathrm{Fe}$ SRPAC spectra (black circles) of $\left[\mathrm{Fe}_{2}\left(\mathrm{~S}_{2} \mathrm{C}_{3} \mathrm{H}_{6}\right)(\mathrm{CO})_{6}\right]$ measured (a) off the nuclear resonance energy by $10 \mathrm{meV}$ and (c) its anisotropic part $2 A_{22} G_{22}(t)$ extracted from (a), (b) on the nuclear resonance energy and (d) its anisotropic part $2 A_{22} G_{22}(t)$ extracted from (b). The gray curves are the spectral simulations using the parameters described in the text. The molecular structure of $\left[\mathrm{Fe}_{2}\left(\mathrm{~S}_{2} \mathrm{C}_{3} \mathrm{H}_{6}\right)(\mathrm{CO})_{6}\right]$ is shown as the inset in (a). The experimental geometry is defined in bottom left scheme of Fig. 12.4 with $\theta=0$. 
The ${ }^{57} \mathrm{Fe}$ SRPAC measurements on this model complex were recorded both on the nuclear resonance energy ( $14.4 \mathrm{keV}$ of ${ }^{57} \mathrm{Fe}$ ), and off $10 \mathrm{meV}$ away from the resonance energy at $120 \mathrm{~K}$ (Fig. I2. I0), an exponential decay signal coupled with oscillations can be seen in both spectra. In the off-resonance spectrum, a simple oscillation pattern is observed with the period of $\sim 110 \mathrm{~ns}$. In the on-resonance spectrum, a similar oscillation pattern is observed after $20 \mathrm{~ns}$, while the spectrum before 20 ns shows a dramatic increase in intensity. As pointed in Section I2.2, this intensity increase comes from the $4 \pi$ scattering produced by NFS, and its intensity is proportional to the NFS signal. That explains when the SRPAC signal is recorded off-resonance, no such intensity increase is observed because NFS signal is significantly reduced when the incident energy is tuned away from the nuclear resonance energy.

We first simulated the off-resonance spectrum. To simulate this spectrum that is dominated by electric quadrupole interactions, Eq. (12.10) was used:

$$
I(t)=I_{0} \mathrm{e}^{-t / \tau_{0}}\left\{1-2 A_{22}\left[w G_{22}^{1}(t)+(1-w) G_{22}^{2}(t)\right] P_{2}(\cos \theta)\right\},
$$

where all symbols have the same definition as in Eq. (12.5). Here two iron sites are used, $w$ is the weight factor of the first iron site. $A_{22}$ is the anisotropy coefficient, in case of ${ }^{57} \mathrm{Fe}, A_{22}=0.25$ theoretically [12]. In the real experimental data, due to the averaging effect from the finite size of detectors and samples, $A_{22}$ is actually the effective anisotropy coefficient, and usually the value is smaller than the theoretical values. The angular dependence of the SRPAC intensity is defined by the Legendre polynomial $P_{2}(\cos \theta)$. It has its maximum value, I, along the direction of the magnetic polarization of the incoming radiation, which is perpendicular to the storage ring plane, here $\theta$ is the angle between the magnetic polarization direction of the incoming synchrotron radiation and the outgoing scattering signal. In our experimental setup, $\theta=0$; therefore, $P_{2}(\cos \theta)=$ I. $G_{22}(t)$ takes the same form as in Eq. (I2.6).

The best fit for the off-resonance spectrum is shown in Fig. 12.10a as the gray solid line. Sometimes, it is also useful to extract the anisotropic part of the scattered intensity, $A_{22} R(t)$, which is also called anisotropy, from the experimental spectrum. The anisotropy spectrum and the spectral simulation are shown in Fig. 12.10c. The weight factor $w$ is fixed at 0.5 in the simulation. For the first iron site, $\Omega=60.95(36) \mathrm{MHz}$, which corresponds to quadrupole splitting $\Delta E_{\mathrm{Q}}=0.835$ (5) $\mathrm{mm} \mathrm{s}^{-1}$, for the second iron site, $\Omega=49.89(4 \mathrm{I}) \mathrm{MHz}$, which corresponds to quadrupole splitting $\Delta E_{\mathrm{Q}}=0.684$ (5) $\mathrm{mm} \mathrm{s}^{-1}$. The result is consistent with the Mössbauer spectrum measured on the same sample at $100 \mathrm{~K}$ (spectrum not shown), which gives two iron sites with quadrupole splitting of 0.85 and $0.70 \mathrm{~mm} \mathrm{~s}^{-1}$, respectively, and also is close to the previously reported Mössbauer spectrum of this model compound measured under $77 \mathrm{~K}$ [67]. Also from this best fit, we obtained the anisotropy coefficient $A_{22}=0.164$, which is smaller than the theoretical value 0.25 , this may due to the angle averaging effect of the size of both detector and sample.

With these parameters in hand, we then started to treat the on-resonance spectrum. It is very difficult to find a proper model to describe the mixture of elastic and inelastic (coherent and incoherent) scattering. The simplest approach, which ignores hyperfine interactions, was derived in Ref. 34. Here, we used a simple exponential decay to simulate the elastic scattering contribution. Then Eq. (12.10) becomes

$$
I(t) \mathrm{e}^{t / \tau_{0}}=I_{0}\left(1+r \mathrm{e}^{-a t / \tau_{0}}\right)\left\{1-2 A_{22}\left[w G_{22}^{1}(t)+(1-w) G_{22}^{2}(t)\right] P_{2}(\cos \theta)\right\},
$$

where $r$ and $a$ are the parameters to describe the elastic scattering contribution. Using Eq. (I 2 . I3), we obtained the best fit for the on-resonance spectrum (Fig. 12. I0b and d) with $r=4.12, a=16.32$, while fixing the other parameters with the values obtained from the off-resonance simulation. The simulation reproduced the spectrum quite well.

The ${ }^{57} \mathrm{Fe}$ SRPAC spectrum of $\mathrm{Fe}_{2}\left(\mathrm{~S}_{2} \mathrm{C}_{3} \mathrm{H}_{6}\right)(\mathrm{CO})_{6}$ and the analysis described above shows the feasibility of using SRPAC in bioinorganic chemistry studies. Similar with NFS, with longer time observation window, more accurate results can be obtained by using SRPAC. To obtain clear SRPAC signal, especially when the time observation window is short (comparable to the nuclear excited-state lifetime), on-resonance measurement should be avoid if possible. However, moving from on-resonance measurement to off-resonance measurement (in our case, $10 \mathrm{meV}$ away from the resonance energy), the count-rate drops by a factor of $>5$, which will be a significant effect if one wants to apply SRPAC to study metal centers in metalloproteins due to the much lower metal concentration in metalloproteins.

12.3.2.2 ${ }^{57} \mathrm{Fe}$ SRPAC of Ni Ferrite $\mathrm{NiFe}_{2} \mathrm{O}_{4}$ is ferrimagnetic material, it exhibit a substantial spontaneous magnetization at room temperature. The spontaneous magnetization disappears above a certain critical temperature $T_{c}$, also called the Curie temperature, and then it becomes paramagnetic. Ni ferrite, like other ferrimagnetic material, has a cubic crystal structure, $\mathrm{Ni}(\mathrm{II})$ and one of the Fe(III) ions occupy the octahedral sites, and the other Fe(III) ions occupy the 


\section{FIGURE 12.11}

(a) ${ }^{57}$ Fe SRPAC spectrum of ${ }^{57}$ Fe-enriched Ni ferrite (black circles) and (b) the spectrum after eliminating the exponential decay (black circles). The gray curves are spectral simulations using the parameters described in the text. The experimental setup is defined in the bottom left scheme of Fig. 12.4 with $\theta=0$ and the applied magnetic field $(0.3 \mathrm{~T})$ aligned along $x$-axis, which was the electric field direction of the incident $\mathrm{X}$-rays.

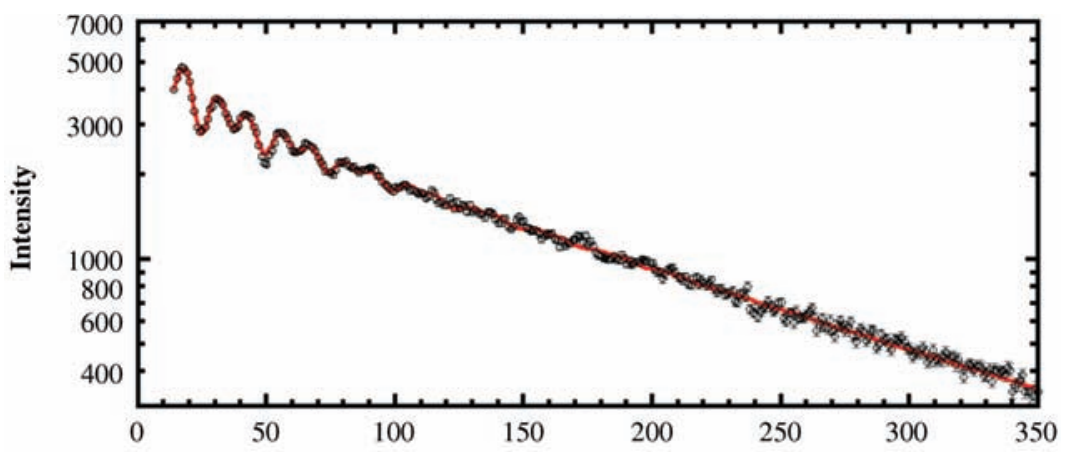

(a) Time (ns)

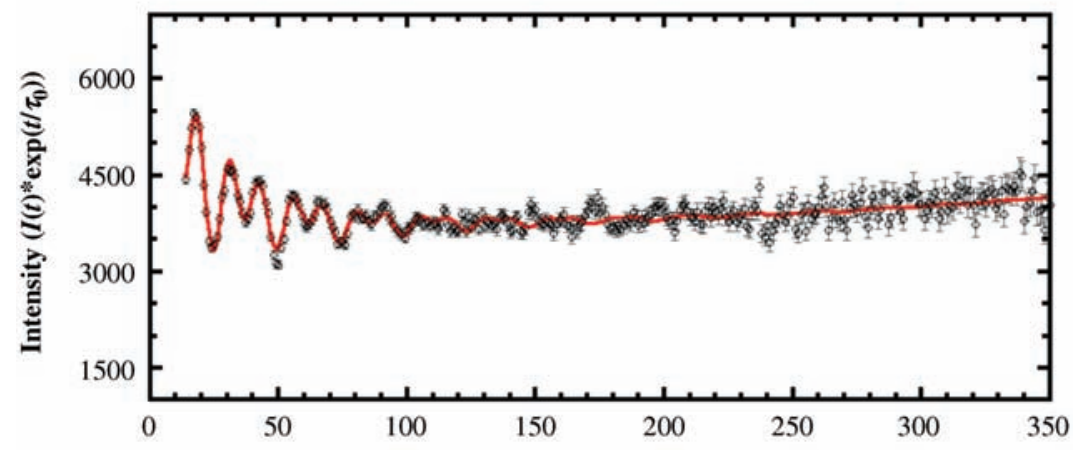

(b) Time (ns)

tetrahedral sites. The different synthetic methods can generate slightly different magnetic hyperfine interactions in $\mathrm{Ni}$ ferrite. In general, the two Fe(III) sites have slightly different internal magnetic fields, but both are close to 50 T [68].

Figure 12.I la shows the ${ }^{57} \mathrm{Fe}$ SRPAC spectrum of Ni ferrite measured $18 \mathrm{meV}$ away from the nuclear resonance energy with $0.3 \mathrm{~T}$ external magnetic field applied along the electric polarization direction of the incoming photon. Different from the quadrupole splittings observed in $\mathrm{Fe}_{2}\left(\mathrm{~S}_{2} \mathrm{C}_{3} \mathrm{H}_{6}\right)(\mathrm{CO})_{6}$, the oscillations observed in ${ }^{57} \mathrm{Fe}$ SRPAC spectrum of $\mathrm{Ni}$ ferrite is due to the magnetic splittings because Ni ferrite shows spontaneous magnetization under room temperature. The initial simulation of the spectrum indicated that both models described by Eqs. (12.7) and (12.8) in Section 12.2 cannot fit the experimental data. A close look at the data revealed three unique features of the spectrum: the incomplete periodicity of the beats, which comes from the incomplete alignment of the magnetic moments in the sample toward the external magnetic field; the damping of the beats with time, which can be explained by distribution of the magnetic field in the sample; and the slight oscillation of the baseline, which is most likely due to the $4 \pi$ solid angle scattering produced by NFS. The theoretical models describing these three unique features have been incorporated in the final model for fitting the experimental data (for detailed discussion, please refer to Ref. 69), which is

$$
\begin{aligned}
& I(t)=I_{0} \mathrm{e}^{-t / \tau_{0}}\left(1+r \mathrm{e}^{-a t / \tau_{0}}\right)\left(1-2 A_{22} R(t)\right)+b, \\
& R(t)=C_{0}+C_{1} \mathrm{e}^{-\Gamma t / 2} \cos (\Omega t)+C_{2} \mathrm{e}^{-\Gamma t} \cos (2 \Omega t),
\end{aligned}
$$

and

$$
\begin{aligned}
& C_{0}=\left(245-22 \cos \theta_{0}+16 \cos 2 \theta_{0}+54 \cos 3 \theta_{0}+27 \cos 4 \theta_{0}\right) / 1280 \\
& C_{1}=\left(105-46 \cos \theta_{0}-32 \cos 2 \theta_{0}-18 \cos 3 \theta_{0}-9 \cos 4 \theta_{0}\right) / 320 \\
& C_{2}=\left(615+206 \cos \theta_{0}+112 \cos 2 \theta_{0}+18 \cos 3 \theta_{0}+9 \cos 4 \theta_{0}\right) / 1280
\end{aligned}
$$

where $I_{0}$ is the intensity of the nuclear fluorescence in the entire solid angle at zero time, $A_{22}$ is the anisotropy coefficient, $b$ is the background, $r$ and $a$ describe the elastic scattering contributions, $\Omega$ is the Larmor frequency of the magnetic splitting measured in natural linewidth $\Gamma_{0}$ (in case of ${ }^{57} \mathrm{Fe}, \Gamma_{0}=4.66 \mathrm{neV}$ ), $\Gamma$ is the FWHM of the magnetic splitting 
distribution measured also in $\Gamma_{0} . \theta_{0}$ describes the misalignment of the magnetic moments in the sample toward the external magnetic field.

The best fit using Eq. (12.12) is shown in Fig. 12.I I a along with the spectrum after eliminating the exponential decay (Fig. 12.I lb). The parameters used for fitting are total intensity $I_{0}$, background $b, r$, and $a$, anisotropy coefficient $A_{22}$, Larmor frequency of the magnetic splitting $\Omega$, FWHM of the magnetic splitting distribution $\Gamma$, and the opening angle of the cone distribution of the directions of magnetic moments $\theta_{0}$. The corresponding values obtained from the best fit are $I_{0}=3932(70) ; b=42(20) ; r=0.58(9) ; a=7.2(9) ; A_{22}=0.22(I)$-which is a little bit less than the theoretical value 0.25 , this is due the angle average over size of both the detector and the sample; $\Omega=36.07(10) \Gamma_{0}$, which corresponds to the hyperfine field of $50.3(\mathrm{I}) \mathrm{T} ; \Gamma=3.4 \Gamma_{0}$, which corresponds to FWHM of the magnetic field distribution $4.9 \mathrm{~T} ; \theta_{0}=35(\mathrm{I})^{\circ}$, which corresponds to the relative magnetization $M / M_{\text {sat }}=0.91$.

To examine the validity of this fitting, a room temperature zero-field Mössbauer spectrum of this sample was measured. The ${ }^{57} \mathrm{Fe}$ Mössbauer spectrum (spectrum not shown) revealed two iron sites with isomer shifts $\delta_{1}=0.27$ $\mathrm{mm} \mathrm{s}^{-1}, \delta_{2}=0.35 \mathrm{~mm} \mathrm{~s}^{-1}$, internal magnetic fields $B_{\text {intl }}=48.7 \mathrm{~T}, B_{\text {int } 2}=52.0 \mathrm{~T}$, and linewidth $\Gamma_{1}=0.41 \mathrm{~mm} \mathrm{~s}, \Gamma_{2}$ $=0.38 \mathrm{~mm} \mathrm{~s}^{-1}$. This result is consistent with the reported Mössbauer result on Ni ferrite [70]. The internal magnetic field obtained by the SRPAC simulation is equal to the average internal magnetic field values obtained by Mössbauer spectroscopy. The $\sim 0.4 \mathrm{~mm} \mathrm{~s}^{-1}$ linewidth for both iron sites from the Mössbauer simulation are relatively broad (for our current Mössbauer spectrometer setup, a well prepared sample gives $0.25-0.30 \mathrm{~mm} / \mathrm{s}$ linewidth). This broadening can be explained by the distribution of the internal magnetic field. By introducing this distribution into the Mössbauer simulation, we obtained the FWHM of the magnetic splitting distribution to be $\sigma\left(B_{\text {int }}\right)=3 \mathrm{~T}$, which confirms the distribution model we used in our SRPAC simulations.

The complicated ${ }^{57} \mathrm{Fe}$ SRPAC spectrum of Ni ferrite was quite unexpected for us at the beginning. However, by including partial alignment of the nuclear magnetic moments, distribution of internal magnetic fields and the elastic scattering contribution into the theoretical model, we reproduced the spectrum very well, and the result is consistent with our Mössbauer data. This indicates that SRPAC can reveal very detailed hyperfine interactions.

12.3.2.3 ${ }^{61} \mathrm{Ni}$ SRPAC of Ni Ferrite The real difficulty for Mössbauer spectroscopy is to study Mössbauer isotopes with high transition energy because of the short lifetime of radioactive source and small Lamb-Mössbauer factor $f_{L M}$. In the case of ${ }^{61} \mathrm{Ni}$, the radioactive source used $\left({ }^{61} \mathrm{Co}\right)$ only has $99 \mathrm{~min}$ of lifetime, and $f_{\mathrm{LM}}$ is $\sim 0.02$ even in the liquid helium temperature. These make the application of Mössbauer spectroscopy to ${ }^{61} \mathrm{Ni}$ extremely difficult. As discussed earlier, SRPAC signal is independent to $f_{\mathrm{LM}}$, and the use of SR eliminates the necessity of radioactive sources. It makes SRPAC an ideal technique to study ${ }^{61} \mathrm{Ni}$. The first ${ }^{61} \mathrm{Ni}$ SRPAC experiment was done by Sergueev et al. on ${ }^{61} \mathrm{Ni}$-enriched $\mathrm{Ni}$ foil to reveal the magnetic hyperfine interactions [18]. Here we report the first application of ${ }^{61} \mathrm{Ni}$ SRPAC to a ${ }^{61} \mathrm{Ni}$-enriched $\mathrm{Ni}$ ferrite to reveal the magnetic hyperfine interactions.

Figure 12.12 shows the ${ }^{61} \mathrm{Ni}$ SRPAC spectra of Ni ferrite recorded at the nuclear resonance energy, $67.4 \mathrm{keV}$, by applying 0.2 T magnetic field in two different directions: aligning along the electric polarization direction of the incoming photon ( $x$-axis in the bottom left scheme of Fig. 12.4), and aligning along the magnetic polarization direction of the incoming photon (z-axis in the bottom left scheme of Fig. 12.4). The detector is always placed below the sample and along the magnetic polarization direction of the incoming photon (z-axis). Both of the spectra mainly show as an exponential decay. The bump at $\sim 15 \mathrm{~ns}$ is an artificial feature caused by detection electronics.

Due to the small Lamb-Mössbauer factor $\mathrm{fLM}_{\mathrm{L}}$, the $4 \pi$ solid angle contribution of coherent elastic scattering observed in ${ }^{57} \mathrm{Fe}$ SRPAC spectra is very small and can be ignored. When the magnetic field is aligned along $x$-axis, according to Eq. (12.8), we can express SRPAC signal as

$$
I(t)=I_{0} \mathrm{e}^{-t / \tau_{0}}\left[1-2 A_{22}\left(1+3 \cos \left(2 \omega_{\mathrm{B}} t-2 \pi\right)\right) / 4\right],
$$

where $\omega_{B}=\left(\left(g_{N}^{e} \mu_{N}\right) / \hbar\right)$ B is the Larmor frequency of the magnetic splitting, $g_{N}^{e}$ is the nuclear excited-state $g$-factor, $\mu_{N}$ is the nuclear Bohr magneton. When the magnetic field is aligned along z-axis, according to Eq. (I2.8), we can express SRPAC signal as

$$
I(t)=I_{0} \mathrm{e}^{-t / \tau_{0}}\left(1-2 A_{22}\right)=I_{0}^{\prime} \mathrm{e}^{-t / \tau_{0}}
$$

We can see that for the second case, all we can observe is the nuclear excited-state exponential decay signal, no SRPAC signal can be observed. 


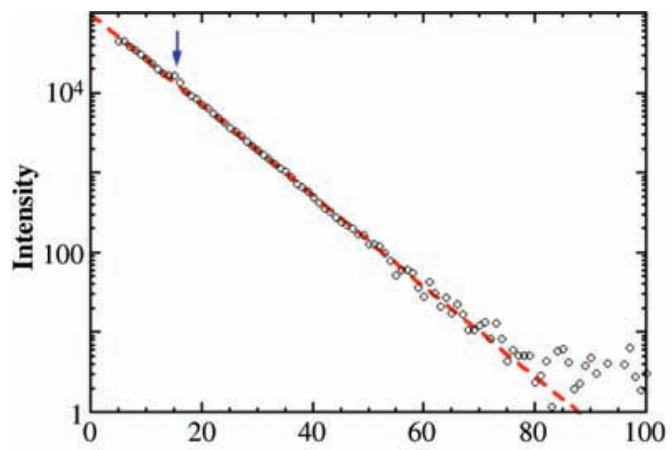

(a) Time (ns)

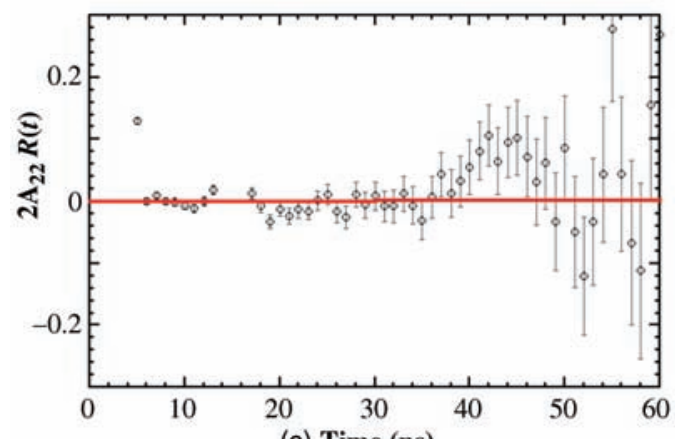

(c) Time (ns)

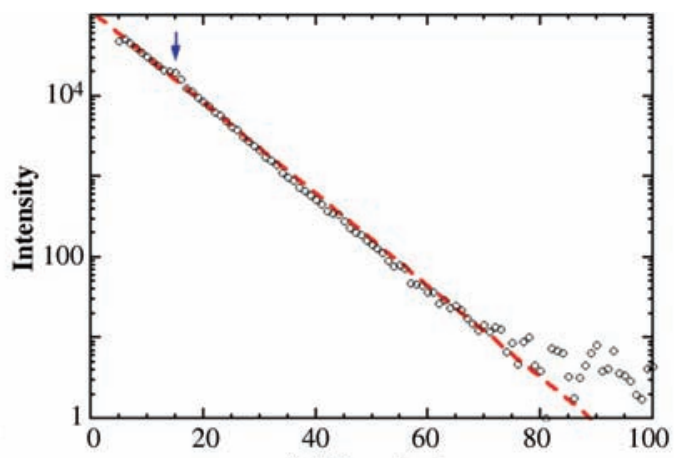

(b) Time (ns)

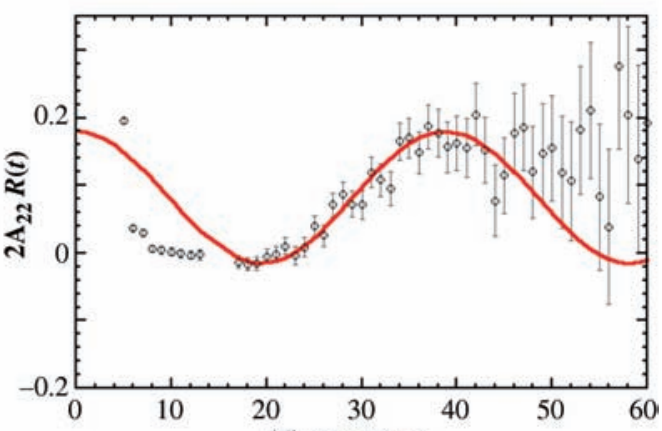

(d) Time (ns)

FIGURE 12.12

${ }^{61} \mathrm{Ni}$ SRPAC of ${ }^{61} \mathrm{Ni}$-enriched $\mathrm{Ni}$ ferrite (black circles) with (a) the applied magnetic field (0.3 T) aligned along z-axis, which is the magnetic polarization direction of the incident $X$-ray, and $(b)$ the applied magnetic field $(0.3 \mathrm{~T})$ aligned along $x$-axis, which is the electric polarization direction of the incident X-ray; (c) the anisotropic part $2 A_{22} R(t)$ extracted from (a); (d) the anisotropic part $2 A_{22} R(t)$ extracted from (b). The experimental setup is defined in the bottom left scheme of Fig. 12.4 with $\theta=0$.

Using Eq. (12.14), we first fit the data with the applied magnetic field aligned along z-axis (Fig. I2.12a) and set $I_{0}^{\prime}$ and the lifetime $\tau_{0}$ as floating parameters. From the fit, we obtain $\tau_{0}=7.65 \pm 0.1 \mathrm{~ns}$, which is consistent with the reported value of $7.7 \pm 0.2 \mathrm{~ns}[7 \mathrm{I}]$. Then we used the nuclear excited-state lifetime obtained from the experiment to extract the anisotropy $A_{22} R(t)$ of the ${ }^{61} \mathrm{Ni}$ SRPAC spectra. The anisotropy of our data are shown in Fig. 12.12c and d. As expected, for the case of aligning the magnetic field along $z$-axis, no oscillation pattern is observed. However, in case of aligning the magnetic field along $x$-axis, a clear cosine oscillation pattern is observed up to $\sim 50 \mathrm{~ns}$ in the anisotropy spectrum. Both anisotropy spectra show a big spike at $15 \mathrm{~ns}$ (the spike has been removed in the anisotropy spectra) and irregular oscillation patterns before $13 \mathrm{~ns}$, the most possible explanation is the influence of artificial signals generated by the detection electronics, such as the reflection of the real signal in some cables.

The best fit for the anisotropy spectrum measured by aligning the magnetic field along $x$-axis gives the oscillation frequency $\omega_{\mathrm{B}}=8 \mathrm{I}(\mathrm{I}) \mathrm{MHz}$ (Fig. 12.12d), which corresponds to an effective magnetic field of $8.8(\mathrm{I}) \mathrm{T}$. Considering the antiparallel alignment nature of the nuclear magnetic moments at $\mathrm{Ni}$ site of $\mathrm{Ni}$ ferrite with the $0.2 \mathrm{~T}$ external magnetic field, the internal magnetic field is $9.0(\mathrm{I}) \mathrm{T}$, which is consistent with the reported $9 . \mathrm{I} \mathrm{T}$ internal magnetic field at Ni site of $\mathrm{Ni}$ ferrite measured by ${ }^{61} \mathrm{Ni}$ Mössbauer spectroscopy [72]. The anisotropy coefficient obtained from this fitting is $A_{22}=0.06(I)$, which is smaller than the theoretical value 0.14 for ${ }^{61} \mathrm{Ni}$. This is most likely due to the angular averaging effect over the size of the detector.

All the above applications indicate that SRPAC is a promising new technique to study hyperfine interactions of Mössbauer isotopes, especially for the isotopes with small Lamb-Mössbauer factors, because of the unique feature of SRPAC - the intensity is independent of the Lamb-Mössbauer factors. However, future developments of this technique has to be done in the direction of increasing the efficiency and the detection area of the APD detector and optimizing the monochromators, especially for the high transition energy Mössbauer isotopes (such as ${ }^{61} \mathrm{Ni}$ with $67.4 \mathrm{keV}$ nuclear resonance energy) so that SRPAC can be regularly applied to protein studies. 


\subsection{SUMMARY AND PROSPECTS}

In this chapter, we presented the applications of SR-based NRS technique to bioinorganic chemistry, particularly NFS and SRPAC techniques. We showed that NFS and SRPAC are capable of revealing detailed hyperfine interaction parameters from the Mössbauer nuclei embedded in rather complicated bioinorganic/biological systems. Furthermore, the use of the third-generation SR in substituting radioactive sources makes NFS and SRPAC better techniques for studying bioinorganic systems than their laboratory-based counterparts, conventional Mössbauer spectroscopy, and TDPAC. The high brightness and highly collimated SR make it possible to probe low-concentration small-volume samples, which is crucial for further studies of more complicated biological systems and reaction intermediates that generally cannot be produced in large quantity and high concentration. In addition to this, the unique time structure of SR and the timediscrimination trick of the detection method make these SR-based techniques low noise level techniques, which further helps in detecting generally weak nuclear resonance signal from biological systems. Moreover, the large tunability of SR opens the possibility to apply NFS and SRPAC technique to broad range of Mössbauer isotopes without the constraint of suitable radioactive sources as normally encountered in conventional Mössbauer spectroscopy and TDPAC. For NFS, the elimination of radioactive sources instantly enhanced the spectral resolution compared with conventional Mössbauer by eliminating the source contribution to the resolution. This is extremely important in resolving spectral features in complicated systems. For SRPAC, the independence of the SRPAC signal to the Lamb-Mössbauer factor makes it a suitable technique to probe Mössbauer isotopes with high nuclear resonance energy, such as ${ }^{61} \mathrm{Ni}$.

The development of NRS is inarguably based on the development of SR source, the X-ray optics, and the detection method. Given the development of SR-based NRS in the past decades and the ongoing development of even brighter next-generation SR sources and X-ray free-electron lasers, we believe that NRS will become essential spectroscopic technique in elucidating geometric and electronic structures of complicated metal containing biological systems.

\section{ACKNOWLEDGMENTS}

We thank Drs. Francis E. Jenney Jr. and Mike W.W. Adams at University of Georgia for providing all the ferredoxin protein samples; Drs. Eric Brecht and John W. Peters for providing us the iron protein of nitrogenase samples; Drs. Karl Fisher and William E. Newton from Virginia Tech for providing us the MoFe protein of nitrogenase samples; and Drs. Christine Boyke and Thomas B. Rauchfuss from University of Illinois for providing us the iron model complexes. We also thank Dr. Ilya Sergueev at the ESRF for the discussions on the SRPAC data simulations, and Dr. Wolfgang Sturhahn at the APS for the use of CONUSS program. This work was funded by NIH grant GM-65440 (S.P.C). Use of SPring-8 was supported by the Japan Synchrotron Radiation Research Institute (JASRI) under Proposals \# 2032LD3-np and I2I5LD3np. Use of the PF-AR was supported by the Photon Factory Program Advisory Committee under Proposal \# 2006 G024.

\section{REFERENCES}

I. G. Lang, Mössbauer spectroscopy of haem proteins, Q. Rev. Biophys. 1970, 3(1), I-60.

2. E. Münck, Mössbauer spectroscopy of proteins: electron carriers, Methods Enzymol. 1978, 54, 346-79.

3. V. Schünemann, H. Winkler, Structure and dynamics of biomolecules studied by Mössbauer spectroscopy, Rep. Prog. Phys. 2000, 63 (3), 263-353.

4. C. Krebs, J.M. Bollinger, Freeze-quench Fe-57 Mössbauer spectroscopy: trapping reactive intermediates, Photosynth. Res. 2009, 102(2-3), 295-304.

5. T. Butz, Nuclear quadrupole interactions studies by time differential perturbed angular correlations of gamma-rays, Z. Naturforsch. A 1996, 5I(5-6), 396-4I0.

6. R. Bauer, Perturbed angular-correlation spectroscopy and its application to metal sites in proteins-possibilities and limitations, $Q$. Rev. Biophys. 1985, I8(I), I-64.

7. L. Hemmingsen, K.N. Sas, E. Danielsen, Biological applications of perturbed angular correlations of $\gamma$-ray spectroscopy, Chem. Rev. 2004, 104, 4027-4061.

8. W. Sturhahn, Nuclear resonant spectroscopy, J. Phys. Condens. Matter 2004, 16, S497-S530.

9. M. Seto, et al., Synchrotron radiation based Mössbauer spectroscopy, Phys. Rev. Lett. 2009, 102(21), 217602. 
10. T. Mitsui, et al., Development of an energy-domain ${ }^{57} \mathrm{Fe}$ Mössbauer spectrometer using synchrotron radiation and its application to ultrahigh-pressure studies with a diamond anvil cell, J. Synchrotron Radiat. 2009, 16(6), 723-729.

II. T.S. Toellner, et al., Synchrotron Mössbauer spectroscopy using high-speed shutters, J. Synchrotron Radiat. 20 I I, I8(2), I83-I88.

12. I. Sergueev, et al., Synchrotron-radiation-based perturbed angular correlations used in the investigation of rotational dynamics in soft matter, Phys. Rev. B 2006, 73(2).

13. E. Gerdau, H. de Waard, eds., Nuclear Resonant Scattering of Synchrotron Radiation, Vols. 123-124, Hyperfine Interactions/Kluwer Academic Publishers, New York, 1999, p. 879.

14. R. Rüffer, Nuclear resonant scattering into the new millennium, Hyperfine Interact. 2002, I4I(I-4), 83-97.

15. R. Röhlsberger, Coherent elastic nuclear resonant scattering, in Nuclear Condensed Matter Physics with Synchrotron Radiation-Basic Principles, Methodology and Applications, Springer, Berlin, 2004, pp. 67-180.

16. H. Paulsen, et al., Mössbauer studies of coordination compounds using synchrotron radiation, Coord. Chem. Rev. 2005, 249(I-2), 255-272.

I7. C. Strohm, I. Sergueev, U. van Burck, Synchrotron-radiation-based perturbed angular correlations from Sn- I 19, Eur. Phys. Lett. 2008, 8 I (5) 5200I-I-5200I-4.

18. I. Sergueev, et al., Studies of hyperfine interactions in Ni-6I with synchrotron-radiation-based perturbed angular correlations, Phys. Rev. B 2008, 78(2I) 2I4436-I-2|4436-6.

19. W. Sturhahn, E. Gerdau, Evaluation of time-differential measurements of nuclear-resonance scattering of X-rays, Phys. Rev. B I 994, 49(I4), 9285-9294.

20. Y.V. Shvyd'ko, Coherent nuclear resonant scattering of X-rays: time and space picture, Hyperfine Interact. I 999, I23(I-8), 275299.

2I. M. Haas, et al., Nuclear resonant forward scattering of synchrotron radiation by randomly oriented iron complexes which exhibit nuclear Zeeman interaction, Phys. Rev. B 1997, 56(2I), I4082-I4088.

22. Y.V. Shvyd'ko, et al., Hybrid beat in nuclear forward scattering of synchrotron radiation, Phys. Rev. B 1998, 57(6), 3552-356I.

23. Y.V. Shvyd'ko, U. van Burck, Hybrid forms of beat phenomena in nuclear forward scattering of synchrotron radiation, Hyperfine Interact. 1999, I 23(I-8), 5II-527.

24. U. Vanburck, et al., Nuclear forward scattering of synchrotron radiation, Phys. Rev. B 1992, 46(10), 6207-62II.

25. I. Sergueev, Nuclear resonant scattering for the study of dynamics of viscous liquids and glasses, Ph.D. thesis, 2003.

26. H.E. Mahnke, Introduction to PAC/PAD, Hyperfine Interact. I989, 49(I-4), 77-I02.

27. E.E. Alp, et al., Nuclear resonant scattering beamline at the advanced photon source, Hyperfine Interact. 1994, 90(I-4), 323-334.

28. R. Rüffer, A.I. Chumakov, Nuclear resonance beamline at ESRF, Hyperfine Interact. I996, 97-8(I-4), $589-604$.

29. Y. Yoda, et al., Nuclear resonant scattering beamline at SPring-8, Nucl. Instrum. Methods Phys. Res. A 200 I, 467, 7I5-7I8.

30. R. Röhlsberger, Methods and instrumentation, in Nuclear Condensed Matter Physics with Synchrotron Radiation-Basic Principles, Methodology and Applications, Springer, Berlin, 2004, pp. 37-65.

3I. A. Chumakov, W. Sturhahn, Experimental aspects of inelastic nuclar resonance scattering, Hyperfine Interact. I 999, I23(I-8), 78I808.

32. S. Kishimoto, Avalanche photodiodes as fast X-ray detectors, J. Synchrotron Radiat. 1998, 5, 275-279.

33. W. Sturhahn, Phase problem in synchrotron Mossbauer spectroscopy, Phys. Rev. B 200I, 63(9).

34. G.V. Smirnov, V.G. Kohn, Theory of nuclear resonant scattering of synchrotron-radiation in the presence of diffusive motion of nuclei, Phys. Rev. B I 995, 52(5), 3356-3365.

35. A.Q.R. Baron, et al., Single-nucleus quantum beats excited by synchrotron radiation, Europhys. Lett. 1996, 34(5), 33 I-336.

36. D.C. Johnson, et al., Structure, function, and formation of biological iron-sulfur clusters, Ann. Rev. Biochem. 2005, 74, 247-28I.

37. H. Beinert, R.H. Holm, E. Münck, Iron-sulfur clusters: Nature's modular, multipurpose structures, Science 1997, 277(5326), 653-659.

38. J. Meyer, Iron-sulfur protein folds, iron-sulfur chemistry, and evolution, J. Biol. Inorg. Chem. 2008, I3(2), I57-I70.

39. I. Bertini, et al., Browsing gene banks for $\mathrm{Fe}_{2} \mathrm{~S}_{2}$ ferredoxins and structural modeling of 88 plant-type sequences: an analysis of fold and function, Proteins 2002, 46(I), II0-127.

40. K. Fukuyama, Structure and function of plant-type ferredoxins, Photosynth. Res. 2004, 8I(3), 289-30I.

4I. H. Lange, et al., A mitochondrial ferredoxin is essential for biogenesis of cellular iron-sulfur proteins, Proc. Natl. Acad. Sci. USA 2000, 97(3), 1050-1055.

42. J. Armengaud, C. Meyer, Y. Jouanneau, A [2Fe-2S] ferredoxin ( $\mathrm{FdVI})$ is essential for growth of the photosynthetic bacterium Rhodobacter capsulatus, J. Bacteriol. 1997, I79(10), 3304-3309.

43. G. Sainz, et al., Structure of a [2Fe-2S] ferredoxin from Rhodobacter capsulatus likely involved in Fe-S cluster biogenesis and conformational changes upon reduction, J. Biol. Inorg. Chem. 2006, II (2), 235-246. 
44. W.R. Dunham, et al., 2-Iron ferredoxins in spinach, parsley, pig adrenal cortex, Azotobacter vinelandii, and Clostridium pasteurianum — studies by magnetic field Mössbauer spectroscopy. 2, Biochim. Biophys. Acta I97I, 253(I), I34.

45. E. Münck, et al., Mössbauer parameters of putidaredoxin and its selenium analog, Biochemistry I972, I I (5), 855.

46. R.E. Anderson, et al., Nature of iron sulfur cluster in a deuterated algal ferredoxin, Biochim. Biophys. Acta 1975, 408(3), 306-3 I8.

47. B.A.S. Barata, et al., Mössbauer study of the native, reduced and substrate-reacted Desulfovibrio gigas aldehyde oxidoreductase, Eur. J. Biochem. 1992, 204(2), 773-778.

48. S.G. Lloyd, et al., Functional necessity and physicochemical characteristics of the [2Fe-2S] cluster in mammalian ferrochelatase, J. Am. Chem. Soc. 1996, II8(4I), 9892-9900.

49. R.E. Duderstadt, et al., Effects of mutations in aspartate 14 on the spectroscopic properties of the $\left[\mathrm{Fe}_{3} \mathrm{~S}_{4}\right]^{+, 0}$ clusters in Pyrococcus furiosus ferredoxin, Biochemistry 1999, 38, 10585-10593.

50. P.S. Brereton, et al., Effect of iron-sulfur cluster environment in modulating the thermodynamic properties and biological function of ferredoxin from Pyrococcus furiosus, Biochemistry 1998, 37, 735I-7362.

5I. Z.H. Zhou, M.W.W. Adams, Site-directed mutations of the 4Fe-ferredoxin from the hyperthermophilic archaeon Pyrococcus furiosus: role of the cluster-coordinating aspartate in physiological electron transfer reactions, Biochemistry 1997, 36(36), 10892-10900.

52. P. Middleton, et al., Interpretation of Mössbauer-spectra of 4-iron ferredoxin from Bacillus stearothermophilus, Eur. J. Biochem. I 978 , $88(I),|35-| 4 \mid$.

53. J.M. Moulis, et al., Unusual features in EPR and Mössbauer-spectra of the 2[4Fe-4Se]+ ferredoxin from Clostridium pasteurianum, J. Biol. Chem. 1984, 259(I8), I396-1402.

54. P. Auric, et al., Analysis of the high-spin states of the $2[4 \mathrm{Fe}-4 \mathrm{Se}]+$ ferredoxin from Clostridium pasteurianum by Mössbauer spectroscopy, Biochem. J. 1987, 242(2), 525-530.

55. B.K. Burgess, D.J. Lowe, Mechanism of molybdenum nitrogenase, Chem. Rev. 1996, 96(7), 2983-30II.

56. P.A. Lindahl, et al., Mössbauer, EPR, and magnetization studies of the Azotobacter vinelandii Fe protein-evidence for a [4Fe-4S]+ cluster with spin $S=3 / 2$, J. Biol. Chem. 1985, 260(20), II60-II73.

57. P.A. McLean, et al., Isotopic hybrids of nitrogenase-Mössbauer study of MoFe protein with selective Fe-57 enrichment of the Pcluster, J. Biol. Chem. 1987, 262(27), I2900-12903.

58. B.E. Smith, G. Lang, Mössbauer spectroscopy of nitrogenase proteins from Klebsiella pneumoniae—structural assignments and mechanistic conclusions, Biochem. J. 1974, 137(2), 169-180.

59. E. Münck, et al., Nitrogenase 8. Mössbauer and EPR spectroscopy-MoFe protein component from Azotobacter vinelandii OP, Biochim. Biophys. Acta 1975, 400(I), 32-53.

60. S.J. Yoo, et al., Mössbauer study of the MoFe protein of nitrogenase from Azotobacter vinelandii using selective ${ }^{57} \mathrm{Fe}$ enrichment of the M-centers, J. Am. Chem. Soc. 2000, I22(20), 4926-4936.

6I. B.H. Huynh, et al., Nitrogenase 12. Mössbauer studies of the MoFe protein from Clostridum pasteurianum W5, Biochim. Biophys. Acta 1980, 623(I), 124-138.

62. B.H. Huynh, E. Münck, W.H. Ormejohnson, Nitrognease-XI-Mössbauer studies on the cofactor centers of the MoFe protein from Azotobacter vinelandii-OP, Biochim. Biophys. Acta 1979, 576(I), 192-203.

63. W. Sturhahn, CONUSS and PHOENIX: evaluation of nuclear resonant scattering data, Hyperfine Interact. 2000, 125, I49-I72.

64. Y.V. Shvyd'ko, MOTIF: evaluation of time spectra for nuclear forward scattering, Hyperfine Interact. 2000, I25(I-4), I73-I88.

65. E.J. Lyon, et al., Coordination sphere flexibility of active-site models for Fe-only hydrogenase: studies in intra- and intermolecular diatomic ligand exchange, J. Am. Chem. Soc. 200 I, I23(14), 3268-3278.

66. J.C. Fontecilla-Camps, et al., Structure/function relationships of [NiFe]- and [FeFe]-hydrogenases (vol. I07, p. 4273, 2007), Chem. Rev. 2007, 107, 54II-54II.

67. A. Winter, L. Zsolnai, G. Huttner, Dinuclear and trinuclear carbonylairon complexes containing I,2-dithiolato and I,3-dithiolato bridging ligands, Z. Naturforsch. B 1982, 37(I I), 1430-1436.

68. J. Chappert, R.B. Frankel, Mössbauer study of ferrimagnetic ordering in nickel ferrite and chromium-substituted nickel ferrite, Phys. Rev. Lett. 1967, 19(10), 570.

69. Y. Guo, Initial studies on iron sulfur proteins and related model compounds via 57Fe and 6INi synchrotron radiation based perturbed angular correlations (SRPAC), in nuclear resonant scattering on nitrogenase, hydrogenase and model systems, Ph.D. thesis, 2009.

70. M.A.F. Ramalho, et al., X-ray diffraction and Mössbauer spectra of nickel ferrite prepared by combustion reaction, J. Mater. Sci. 2007, 42, 3603-3606.

7I. M.R. Bhat, Nuclear data sheets for $A=6$ I, Nucl. Data Sheets 1999, 88(3), 4I7-532.

72. P. Gutlich, et al., Ni-6I Mössbauer-spectroscopy of magnetic hyperfine interaction in nickel spinels, J. Chem. Phys. I984, 8I(3), |396-|405. 